\title{
NEW SETS OF INDEPENDENT POSTULATES FOR THE ALGEBRA OF LOGIC, WITH SPECIAL REFERENCE TO WHITEHEAD AND RUSSELL'S PRINCIPIA MATHEMATICA*
}

\author{
BY \\ EDWARD V. HUNTINGTON
}

\section{INTRODUCTION}

Three sets of independent postulates for the algebra of logic, or Boolean algebra, were published by the present writer in 1904. The first set, based on the treatment in Whitehead's Universal Algebra, is expressed in terms of $(K$, $+, \mathrm{X})$, where $K$ is a class of undefined elements, $a, b, c, \cdots$, and $a+b$ and $a \times b$ are the results of two undefined binary operations. The second set is expressed in terms of $(K,<)$, where $a<b$ is an undefined binary relation be-

* Presented to the Society, December 28, 1931, and September 2 and October 29, 1932; received by the editors June 27, 1932. A brief bibliography of postulates for Boolean algebra, which makes no pretence of being complete, is as follows:

E. Schröder, Algebra der Logik. Leipzig, Teubner, 1890.

A. N. Whitehead, Universal Algebra. Cambridge University Press, 1898.

E. V. Huntington, Sets of independent postulates for the algebra of logic. These Transactions, vol. 5 (1904), pp. 288--309.

E. Schröder, Abriss der Algebra der Logik. Leipzig, Teubner, 1909-1910.

A. Del Re, Sulla indipendenza dei postulati della logica. Rendiconto, Accademia delle Scienze, Naples, (3), vol. 17 (1911), pp. 450-458.

H. M. Sheffer, $A$ set of five independent postulates for Boolean algebra, with application to logical constants. These Transactions, vol. 14 (1913), pp. 481-488.

B. A. Bernstein, $A$ complete set of postulates for the logic of classes expressed in terms of the operation "exception," and a proof of the independence of a set of postulates due to Del Re. University of California Publications on Mathematics, vol. 1 (1914), pp. 87-96.

L. L. Dines, Complete existential theory of Sheffer's postulates for Boolean algebras. Bulletin of the American Mathematical Society, vol. 21 (1915), pp. 183-188.

B. A. Bernstein, $A$ set of four independent postulates for Boolean algebra. These Transactions, vol. 17 (1916), pp. 50-51.

B. A. Bernstein, $A$ simplification of the Whitehead-Huntington set of postulates for Boolean algebras. Bulletin of the American Mathematical Society, vol. 22 (1916), pp. 458-459.

J. G. P. Nicod, A reduction in the number of the primitive propositions of logic. Proceedings of the Cambridge Philosophical Society, vol. 19 (1917), pp. 32-41.

N. Wiener, Certain formal invariances in Boolean algebras. These Transactions, vol. 18 (1917), pp. 65-72.

C. I. Lewis, A Survey of Symbolic Logic. University of California Press, 1918.

H. M. Sheffer, Review of C. I. Lewis's "A Survey of Symbolic Logic." American Mathematical Monthly, vol. 27 (1920), pp. 309-311. 
tween the elements $a$ and $b$. The third set is expressed in terms of $(K,+)$, or, if one prefers, in terms of $(K, X)$.

If the class $K$ is finite, it is well known that the number of elements must be some power of 2 ; and any class consisting of $2,4,8,16, \cdots$ elements can be made into a Boolean algebra by properly defining + and $X$.

Every Boolean algebra contains a "zero element," $z$, such that $a+z=a$, and a "universe element," $v$, such that $a \times v=a$; and each element $a$ determines an element $a^{\prime}$, called the "negative" of $a$, such that $a+a^{\prime}=U$ and $a \times a^{\prime}=z$.

In 1913, H. M. Sheffer published a set of postulates for the same algebra expressed in terms of $(K, \mid)$, where the "stroke," |, represents another binary operation, called "rejection," such that $a \mid b=(a+b)^{\prime}$.

A. N. Whitehead and B. Russell, Principia Mathematica, second edition. Cambridge University Press, vol. 1, 1925.

H. M. Sheffer, Review of "Principia Mathematica." Isis, Quarterly organ of the History of Science Society, vol. 8(I) (1926), pp. 226-231.

Paul Bernays, Axiomatische Untersuchung des Aussagen-Kalküls der "Principia Mathematica." Mathematische Zeitschrift, vol. 25 (1926), pp. 305-320.

B. A. Bernstein, Sets of postulates for the logic of propositions. These Transactions, vol. 28 (1926), pp. $472-478$.

D. Hilbert and W. Ackermann, Grundzüge der theoretischen Logik. Berlin, 1928.

Alfred Tarski, Fundamentale Begriffe der Methodologie der deduktiven Wissenschaften. I. Monatshefte für Mathematik und Physik, vol. 37 (1930), pp. 1-44.

Kurt Gödel, Die Vollständigkeit der Axiome des logischen Funktionenkalküls. Monatshefte, vol. 37 (1930), pp. 349-360.

Kurt Gödel, Über formal unentscheidbare Sätze der Principia Mathematica und verwandter Systeme. I. Monatshefte, vol. 38 (1931), pp. 173-198.

J. Lukasiewicz and A. Tarski, Untersuchungen über den Aussagenkalkül. Comptes Rendus des Séances de la Société des Sciences et des Lettres de Varsovie, vol. 23 (1930), Class III, pp. 1-21.

J. Lukasiewicz, Philosophische Bemerkungen zu mehrwertigen Systemen des Aussagenkalküls. Ibid., pp. 51-77.

A. Heyting, Die formalen Regeln der intuitistischen Logik. Sitzungsberichte der preussischen Akademie der Wissenschaften (Berlin), Jahrgang 1930, Physikalisch-Mathematische Klasse, pp. 42-56; 57-71; 158-169.

B. A. Bernstein, Whitehead and Russell's theory of deduction as a mathematical science. Bulletin of the American Mathematical Society, vol. 37 (1931), pp. 480-488.

J $\phi$ rgen J $\phi$ rgensen, $A$ Treatise of Formal Logic. Oxford University Press, 3 vols., 1931.

E. V. Huntington, $A$ new set of independent postulates for the algebra of logic with special reference to Whitehead and Russell's Principia Mathematica. (This brief abstract includes the "fourth set" in the present paper and one other set of a different character.) Proceedings of the National Academy of Sciences, vol. 18 (1932), pp. 179-180.

P. Henle, The independence of the postulates of logic. Bulletin of the American Mathematical Society, vol. 38 (1932), pp. 409-414.

B. A. Bernstein, On proposition *4.78 of Principia Mathematica, Bulletin of the American Mathematical Society, vol. 38 (1932), pp. 388-391.

C. I. Lewis and C. H. Langford, Symbolic Logic. New York, The Century Company, 1932. 
In 1914, B. A. Bernstein gave a set in terms of $(K,-)$, where the "-" represents another binary operation called "exception," such that $a-b$ $=a \times b^{\prime}$; and also a set in terms of $(K, \div)$, where the " $\div$ " indicates a binary operation called "adjunction," such that $a \div b=a+b$ '.

In the meantime, the primitive propositions of Section A of the Principia Mathematica (1910) were expressed in terms of a class called the class of "elementary propositions," a binary operation called "disjunction," and a unary operation called "negation"; and Bernstein has recently shown (June, 1931) how these primitive propositions can be expressed in abstract mathematical form in terms of $\left(K,+,^{\prime}\right)$. Since the relation between the theory of the Principia and the theory of Boolean algebra has been the subject of some discussion, it becomes a matter of interest to construct a set of independent postulates for Boolean algebra explicitly in terms of $\left(K,+,{ }^{\prime}\right)$, for comparison with the Principia.

The present paper contains several such sets, numbered in such a way as to avoid confusion with the first, second, and third sets of 1904 .

The fourth set, containing six postulates, appears to be the simplest and most "natural" of all the sets of postulates for Boolean algebra. It contains no "existence" postulate.

The fifth set, suggested by Sheffer's set of 1913, is shorter by one postulate, but appears decidedly more "artificial" than the fourth set.

The sixth set is modeled after the Principia-Bernstein set, with the addition of an extra postulate which proves to be necessary to make the list sufficient for Boolean algebra. This set also appears artificial and complicated in comparison with the fourth set.

All three of these sets are expressed in terms of $\left(K,+,{ }^{\prime}\right)$; but since in all these sets (following the usual mathematical custom) tacit use is made of the equality sign, it is more accurate to say that all these sets are expressed in terms of $\left(K,+,{ }^{\prime},=\right)$.

In the present paper, the rules governing the use of the equality sign are listed in explicit form as Postulates A, B, C, D. Such an explicit statement of the postulates governing the sign $=$ is essential to any satisfactory comparison between Boolean algebra and the Principia.

For, an outstanding feature of the Principia is that no postulates for $=$ are presupposed. The primitive propositions of the Principia do not contain the equality sign, and the development of the theory proceeds without the use of Postulates A, B, C, D. Instead, a symbol $\equiv$ is introduced by definition, and Postulates A, B, C, D (with $\equiv$ written in place of $=$ ) are supposed to be deduced as theorems.

It appears, however, that the desired properties of the sign $\equiv$, as de- 
scribed in the informal part of the Principia, cannot be rigorously deduced from the formal list of primitive propositions and the formal definition of $\equiv$ in the Principia, without the use of some additional postulates.

In Appendix I of the present paper, the connection between a Boolean system $\left(K,+,{ }^{\prime},=\right)$ and the Principia system $\left(K,+,{ }^{\prime}, \equiv\right)$ is explained; and in Appendix II a revised list of primitive propositions for the Principia is given.

The resulting expression of the Principia's system in strictly postulational form is believed to be free from the objections which might be raised against any formulation (like Bernstein's of June, 1931) which pre-supposes the use of the equality sign.

The new set of postulates for the Principia are shown to be "consistent" and "independent" by the same methods that apply to any other set of mathematical postulates.

\section{THE FIRST SET (1904)}

For convenience of reference, the postulates of the "first set" for Boolean algebra, which are expressed in terms of $K,+, \times$, are here reproduced, in abbreviated form, with the original numbering. (The original $\wedge, \vee$, and $\bar{a}$ are here replaced by $z, U$, and $a^{\prime}$; and the circles around the + and $X$ are omitted.)

Ia. If $a$ and $b$ are in the class $K$, then $a+b$ is in the class $K$.

Ib. If $a$ and $b$ are in the class $K$, then $a b$ is in the class $K$.

IIa. There is an element $z$ such that. $a+z=a$ for every element $a$.

IIb. There is an element $v$ such that $a_{U}=a$ for every element $a$.

IIIa. $a+b=b+a$.

IIIb. $a b=b a$.

IVa. $a+b c=(a+b)(a+c)$.

IVb. $a(b+c)=a b+a c$.

V. For each element $a$ there is an element $a^{\prime}$ such that $a+a^{\prime}=U$ and $a a^{\prime}=z$.

VI. There are at least two distinct elements in the class $K$.

From these postulates the following theorems are deduced in the paper cited.

VIIa. The $z$ in IIa is unique.

VIIIa. $a+a=a$.

VIIb. The $v$ in IIb is unique.

IXa. $a+v=v$.

VIIIb. $a a=a$.

$\mathrm{Xa} \cdot a+a b=a$.

IXb. $a z=z$.

$\mathrm{XI}$. The element $a^{\prime}$ in $\mathrm{V}$ is uniquely determined by $a$.

XIIa. $a+b=\left(a^{\prime} b^{\prime}\right)^{\prime}$.

XIIb. $a b=\left(a^{\prime}+b^{\prime}\right)^{\prime}$. 
XIIIa. $(a+b)+c=a+(b+c) . \quad$ XIIIIb. $(a b) c=a(b c)$.

XIV. The relation $a<b$ is defined by any one of the following equations: $a+b=b ; a b=a ; a^{\prime}+b=v ; a b^{\prime}=z$.

Concerning the relation < we have the following theorems, 2.1-2.9, which correspond to the postulates 1-9 of the "second set" in the paper of 1904.

2.1. $a<a$.

2.2. If $a<b$ and $b<a$, then $a=b$.

2.3. If $a<b$ and $b<c$, then $a<c$.

2.4. $z<a$ (where $z$ is the element in IIa and VIIa).

2.5. $a<v$ (where $v$ is the element in IIb and VIIb).

2.6. $a<a+b$; and if $a<y$ and $b<y$, then $a+b<y$.

2.7. $a b<a$; and if $x<a$ and $x<b$, then $x<a b$.

2.8. If $x<a$ and $x<a^{\prime}$, then $x=z$; and if $a<y$ and $a^{\prime}<y$, then $y=v$.

2.9. If $a<b^{\prime}$ is false, then there is at least one element $x$, distinct from $z$, such that $x<a$ and $x<b$.

\section{EXAMPLES OF BOOLEAN ALGEBRAS*}

The most familiar example of a Boolean algebra is the following:

$K=$ the class of regions in a square (including the null region, and the whole square);

$a+b=$ the smallest region which includes both $a$ and $b$;

$a^{\prime}=$ the region complementary to $a$ with respect to the square;

$a b=$ the region common to $a$ and $b$.

Here the relation $a<b$ means " $a$ is included in $b . "$

Another interesting example is the following, given by H. M. Sheffer in his review of C. I. Lewis's $A$ Survey of Symbolic Logic (American Mathematical Monthly, vol. 27 (1920), p. 310):

$K=\mathrm{a}$ class of eight numbers, $1,2,3,5,6,10,15,30$;

$a+b=$ the least common multiple of $a$ and $b$;

$a^{\prime}=30 / a$;

$a b=$ the highest common factor of $a$ and $b$.

Here the relation $a<b$ means " $a$ is a factor of $b$."

Or, in general, let $K=$ the class of $2^{m}$ numbers which are the factors of any Boolean integer, $v$ ("Boolean integer" being the name given by Sheffer to any integer which contains no square factor); with $a+b, a^{\prime}$, and $a b$ defined as illustrated above for the case $U=30$.

Another example for eight elements is the following:

* The name Boolean algebra (or Boolean "algebras") for the calculus originated by Boole, extended by Schröder, and perfected by Whitehead seems to have been first suggested by Sheffer, in 1913. 
$K=\mathrm{a}$ class of eight numbers: $0 ; 2,3,4 ; 23,24,34$; and $234(=U)$;

$a+b, a^{\prime}$ and $a b$ being defined as in the accompanying tables.

\begin{tabular}{|c|c|c|c|c|c|c|c|c|c|c|c|c|c|c|c|c|c|}
\hline+ & 0 & 234 & 2 & 34 & 3 & 24 & 4 & 23 & 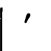 & $\times$ & 02 & & 2 & 34 & 3 & 24 & 423 \\
\hline 0 & 0 & 234 & 2 & 34 & 3 & 24 & 4 & 23 & 234 & 0 & & 0 & 0 & 0 & 0 & 0 & 0 \\
\hline 34 & 234 & 234 & 234 & 234 & 234 & 234 & 234 & 234 & 0 & 234 & & 34 & 2 & 34 & 3 & 24 & $\begin{array}{ll}423 \\
\end{array}$ \\
\hline 2 & 2 & 234 & 2 & 234 & 23 & 24 & 24 & 23 & 34 & 2 & & & 2 & 0 & 0 & 2 & 0 \\
\hline 34 & 34 & 234 & 234 & 34 & 34 & 234 & 34 & 234 & 2 & 34 & 0 & & 0 & 34 & 3 & 4 & 4 \\
\hline 3 & 3 & 234 & 23 & 34 & 3 & 234 & 34 & 23 & 24 & 3 & 0 & & 0 & 3 & 3 & 0 & 0 \\
\hline 24 & 24 & 234 & 24 & 234 & 234 & 24 & 24 & 234 & 3 & 24 & 0 & 24 & 2 & 4 & 0 & 24 & 4 \\
\hline 4 & 4 & 234 & 24 & 34 & 34 & 24 & 4 & 234 & 23 & 4 & 0 & & 0 & 4 & 0 & 4 & 4 \\
\hline 23 & 23 & 234 & 23 & 234 & 23 & 234 & 234 & 23 & 4 & 23 & 0 & 23 & 2 & 3 & 3 & 2 & $\begin{array}{ll}0 & 2\end{array}$ \\
\hline
\end{tabular}

It will be observed that the digits in $a+b$ include the digits in $a$ and also the digits in $b$ ( 0 not counting as a digit); and the digits in $a b$ are the digits common to $a$ and $b$. Hence the commutative, associative and distributive laws are seen at once to be true. Also, the numbers 0 and 234 are seen to serve as the elements $z$ and $U$. By the same process, we can readily construct an example for $2^{m}$ elements, where $m$ is any integer.

The tables for four elements are conveniently written as follows:

\begin{tabular}{|c|c|c|c|c|c|}
\hline+ & 0 & 1 & 2 & 3 & \\
\hline 0 & 0 & 1 & 2 & 3 & 1 \\
\hline 1 & 1 & 1 & 1 & 1 & 0 \\
\hline 2 & 2 & 1 & 2 & 1 & 3 \\
\hline 3 & 3 & 1 & 1 & 3 & 2 \\
\hline
\end{tabular}

\begin{tabular}{|c|c|c|c|c|}
\hline$x$ & 0 & 1 & 2 & 3 \\
\hline 0 & 0 & 0 & 0 & 0 \\
\hline 1 & 0 & 1 & 2 & 3 \\
\hline 2 & 0 & 2 & 2 & 0 \\
\hline 3 & 0 & 3 & 0 & 3 \\
\hline
\end{tabular}

(These tables are the same as the upper left hand quarters of the tables for eight elements, the digit 4 being dropped, and the universe element 234 being represented by 1.)

\section{Postulates governing the USE OF THE EQUALity SIGN}

The postulates of the fourth, fifth, and sixth sets are expressed in terms of the undefined concepts $\left(K,+,{ }^{\prime}\right)$, the first two postulates in each set being the following:

Postulate 1. If $a$ and $b$ are in the class $K$, then $a+b$ is in the class $K$; 
Postulate 2. If $a$ is in the class $K$, then $a^{\prime}$ is in the class $K$;

and in each of these sets (following the usual mathematical procedure), the use of the equality sign, =, is taken for granted.

If preferred, however, the equality sign itself may be regarded as an additional undefined concept, provicied suitable postulates are laid down governing its use.

An obvious set of postulates for $=$ is as follows, where $a, b, c, \cdots$ are understood to be elements of the class $K$.

Postulate A. If $a$ is in the class $K$, then $a=a$.

Postulate B. If $a=b$, then $b=a$.

Postulate C. If $a=b$ and $b=c$, then $a=c$.

Postulate D. If $x=y$, then $f(x, a, b, c, \cdots)=f(y, a, b, c, \cdots)$, where $f(x, a, b, c, \cdots)$ is any element of the class $K$ built up from the elements $x, a$, $b, c, \cdots b y$ successive applications of the operators + and ' (see Postulates 1 and 2$)$, and $f(y, a, b, c, \cdots)$ is the element obtained from $f(x, a, b, c, \cdots)$ by writing $y$ in place of $x$ throughout.

If these postulates A, B, C, D are added, the fourth, fifth, and sixth sets of postulates may be said to express Boolean algebra in terms of the four undefined concepts $\left(K,+,{ }^{\prime},=\right)$.

\section{THE FOURTH SET}

The following set of independent postulates for Boolean algebra is expressed in terms of $\left(K,+,{ }^{\prime}\right) . K$ is a class of elements $a, b, c, \cdots ; a+b$ denotes the result of a binary operation called logical addition; and $a^{\prime}$ denotes the result of a unary operation called logical negation. (A trivial preliminary postulate 4.0, demanding that the class $K$ shall contain at least two distinct elements, is assumed without further mention; and in Postulates 4.3-4.6 it is assumed that the indicated combinations are elements of $K$. Also, Postulates $\mathrm{A}, \mathrm{B}, \mathrm{C}, \mathrm{D}$ are assumed without further mention.)

Postulate 4.1. If $a$ and $b$ are in the class $K$, then $a+b$ is in the class $K$. Postulate 4.2. If $a$ is in the class $K$, then $a^{\prime}$ is in the class $K$.

Postulate 4.3. $a+b=b+a$.

Postulate 4.4. $(a+b)+c=a+(b+c)$.

Postulate 4.5. $a+a=a$.

Postulate 4.6. $\left(a^{\prime}+b^{\prime}\right)^{\prime}+\left(a^{\prime}+b\right)^{\prime}=a$.

By aid of the usual definition of $a \times b$ (or $a b$ ), namely:

4.7. Definition. $a b=\left(a^{\prime}+b^{\prime}\right)^{\prime}$,

the last postulate can be thrown into the following more familiar form:

4.8. $a b+a b^{\prime}=a$. 
From 4.6 , by 4.3 , we have $\left(a^{\prime}+b\right)^{\prime}+\left(a^{\prime}+b^{\prime}\right)^{\prime}=a$, whence by 4.2 , $\left(a^{\prime}+b^{\prime}\right)^{\prime}+\left(a^{\prime}+b^{\prime \prime}\right)^{\prime}=a$. But by $4.7,\left(a^{\prime}+b^{\prime}\right)^{\prime}=a b$ and $\left(a^{\prime}+b^{\prime \prime}\right)^{\prime}=a b^{\prime}$. Hence $a b+a b^{\prime}=a$. Conversely, from 4.7 and 4.8 we have $\left(a^{\prime}+b^{\prime}\right)^{\prime}+\left(a^{\prime}+b^{\prime \prime}\right)^{\prime}=a$, whence by 4.10 , below, $\left(a^{\prime}+b^{\prime}\right)^{\prime}+\left(a^{\prime}+b\right)^{\prime}=a$.

The consistency of these postulates is established by the existence of any system $\left(K,+,{ }^{\prime}\right)$ which satisfies them all, as, for example, any one of the examples of Boolean algebra mentioned above.

To establish the equivalence of this fourth set (which is expressed in terms of $K,+,,^{\prime}$ ) and the first set of 1904 (which is expressed in terms of $K,+, \times$ ), we must show (1) that all the postulates of the fourth set are deducible from the postulates of the first set, when $a^{\prime}$ is properly defined in terms of + and $X$; and (2) that all the postulates of the first set are deducible from the postulates of the fourth set, when $a \times b$ is properly defined in terms of + and '.

The first part of the proof is immediately evident from the preceding section.

The second part of the proof is provided by the following theorems which are deduced from Postulates 4.1-4.6, with the aid of the definition of $a \times b$ contained in 4.7.

4.9. $a+a^{\prime}=a^{\prime}+a^{\prime \prime}$.

By 4.6, $[a]+\left[a^{\prime}\right]=\left[\left(a^{\prime}+a^{\prime \prime \prime}\right)^{\prime}+\left(a^{\prime}+a^{\prime \prime}\right)^{\prime}\right]+\left[\left(a^{\prime \prime}+a^{\prime \prime \prime}\right)^{\prime}+\left(a^{\prime \prime}+a^{\prime \prime}\right)^{\prime}\right]$ and $\left[a^{\prime}\right]+\left[a^{\prime \prime}\right]=\left[\left(a^{\prime \prime}+a^{\prime \prime}\right)^{\prime}+\left(a^{\prime \prime}+a^{\prime}\right)^{\prime}\right]+\left[\left(a^{\prime \prime \prime}+a^{\prime \prime}\right)^{\prime}+\left(a^{\prime \prime \prime}+a^{\prime}\right)^{\prime}\right]$. Hence by 4.3 and $4.4, a+a^{\prime}=a^{\prime}+a^{\prime \prime}$.

Alternative proof, using 4.7 and 4.8 in place of 4.6: By 4.8, $a+a^{\prime}=$ $\left(a a^{\prime}+a a^{\prime \prime}\right)+\left(a^{\prime} a^{\prime}+a^{\prime} a^{\prime \prime}\right)$ and $a^{\prime}+a^{\prime \prime}=\left(a^{\prime} a+a^{\prime} a^{\prime}\right)+\left(a^{\prime \prime} a+a^{\prime \prime} a^{\prime}\right)$. Hence by 4.3 and 4.4 (since by 4.3 and $4.7, a b=b a$ ), we have $a+a^{\prime}=a^{\prime}+a^{\prime \prime}$.

4.10. $a^{\prime \prime}=a$.

By 4.6, $\left(a^{\prime \prime \prime}+a^{\prime \prime}\right)^{\prime}+\left(a^{\prime \prime \prime}+a^{\prime}\right)^{\prime}=a^{\prime \prime}$ and $\left(a^{\prime}+a^{\prime \prime \prime}\right)^{\prime}+\left(a^{\prime}+a^{\prime \prime}\right)^{\prime}=a$. But by $4.9, a^{\prime}+a^{\prime \prime}=a^{\prime \prime}+a^{\prime \prime \prime}$. Hence by $4.3, a^{\prime \prime}=a$.

Alternative proof, using 4.7 and 4.8 in place of $4.6: \mathrm{By} 4.8, a^{\prime \prime} a+a^{\prime \prime} a^{\prime}$ $=a^{\prime \prime}$ and $a a^{\prime}+a a^{\prime \prime}=a$. Hence by 4.7 and $4.3,\left(a^{\prime}+a^{\prime \prime \prime}\right)^{\prime}+\left(a^{\prime \prime}+a^{\prime \prime \prime}\right)^{\prime}=a^{\prime \prime}$ and $\left(a^{\prime}+a^{\prime \prime}\right)^{\prime}+\left(a^{\prime}+a^{\prime \prime \prime}\right)^{\prime}=a$. But by $4.9, a^{\prime}+a^{\prime \prime}=a^{\prime \prime}+a^{\prime \prime \prime}$. Hence by 4.3, $a^{\prime \prime}=a$.

4.11. $a+a^{\prime}=b+b^{\prime}$.

Let $x=a+a^{\prime}$ and $y=b+b^{\prime}$. Then by 4.3, 4.6, 4.5, 4.4, 4.9, $y=b^{\prime}+b=$ $b^{\prime}+\left[\left(b^{\prime}+b^{\prime}\right)^{\prime}+\left(b^{\prime}+b\right)^{\prime}\right]=b^{\prime}+\left(b^{\prime \prime}+y^{\prime}\right)=\left(b^{\prime}+b^{\prime \prime}\right)+y^{\prime}=\left(b+b^{\prime}\right)+y^{\prime}=y+y^{\prime}$. But by 4.6 , with 4.3 and 4.4 ,

$$
\begin{aligned}
y+y^{\prime} & =\left[\left(y^{\prime}+x^{\prime \prime}\right)^{\prime}+\left(y^{\prime}+x^{\prime}\right)^{\prime}\right]+\left[\left(y^{\prime \prime}+x^{\prime \prime}\right)^{\prime}+\left(y^{\prime \prime}+x^{\prime}\right)^{\prime}\right] \\
& =\left[\left(x^{\prime \prime}+y^{\prime \prime}\right)^{\prime}+\left(x^{\prime \prime}+y^{\prime}\right)^{\prime}\right]+\left[\left(x^{\prime}+y^{\prime \prime}\right)^{\prime}+\left(x^{\prime}+y^{\prime}\right)^{\prime}\right] .
\end{aligned}
$$


Hence, by $4.6, y=x^{\prime}+x$.

Again, by 4.3, 4.6, 4.5, 4.4, 4.9,

$$
\begin{aligned}
x & =a^{\prime}+a=a^{\prime}+\left[\left(a^{\prime}+a^{\prime}\right)^{\prime}+\left(a^{\prime}+a\right)^{\prime}\right]=a^{\prime}+\left(a^{\prime \prime}+x^{\prime}\right) \\
& =\left(a^{\prime}+a^{\prime \prime}\right)+x^{\prime}=\left(a+a^{\prime}\right)+x^{\prime}=x+x^{\prime} .
\end{aligned}
$$

Hence, by $4.3, x=y$.

4.12. Definition. $v=a+a^{\prime}=$ the "universe" element of the system.

This element $v$ exists, by 4.1 and 4.2 , and is unique, by 4.11. Moreover, by $4.3, v=a^{\prime}+a$.

4.13. Definition. $z=\left(a+a^{\prime}\right)^{\prime}=$ the "zero" element of the system.

This element $z$ exists, by 4.1 and 4.2 , and is unique, by 4.11 . Obviously, $z=U^{\prime}$, where $U$ is the universe element of 4.12 ; and by $4.10, z^{\prime}=v$.

4.14. If $a^{\prime}=b^{\prime}$, then $a=b$. (By 4.2 and 4.10.)

4.15. $z+a=a$.

By 4.6, $\left(a^{\prime}+a^{\prime}\right)^{\prime}+\left(a^{\prime}+a\right)^{\prime}=a$. Hence by 4.5 and $4.3,\left(a^{\prime}\right)^{\prime}+\left(a+a^{\prime}\right)^{\prime}=a$. Hence by 4.10 and $4.12, a+U^{\prime}=a$, whence by 4.13 and $4.3, z+a=a$.

4.16. $a U=a$.

By 4.7, 4.3, 4.13, 4.15, 4.10, $a U=\left(a^{\prime}+U^{\prime}\right)^{\prime}=\left(U^{\prime}+a^{\prime}\right)^{\prime}=\left(z+a^{\prime}\right)^{\prime}=\left(a^{\prime}\right)^{\prime}=a$.

4.17. $a a^{\prime}=z$.

By 4.7, 4.12, 4.13, $a a^{\prime}=\left(a^{\prime}+a^{\prime \prime}\right)^{\prime}=U^{\prime}=z$.

4.18. $a b=b a$. (By 4.7 and 4.3.)

4.19. $(a b) c=a(b c)$.

By 4.7 and 4.10, $(a b) c=\left(a^{\prime}+b^{\prime}\right)^{\prime} c=\left[\left(a^{\prime}+b^{\prime}\right)^{\prime \prime}+c^{\prime}\right]^{\prime}=\left[\left(a^{\prime}+b^{\prime}\right)+c^{\prime}\right]^{\prime}$ and $a(b c)=a\left(b^{\prime}+c^{\prime}\right)^{\prime}=\left[a^{\prime}+\left(b^{\prime}+c^{\prime}\right)^{\prime \prime}\right]^{\prime}=\left[a^{\prime}+\left(b^{\prime}+c^{\prime}\right)\right]^{\prime}$. But these two values are equal by 4.4 .

4.20. $a+b=\left(a^{\prime} b^{\prime}\right)^{\prime}$.

By 4.7 and $4.10, a^{\prime} b^{\prime}=\left(a^{\prime \prime}+b^{\prime \prime}\right)^{\prime}=(a+b)^{\prime}$. Hence by 4.10, $\left(a^{\prime} b^{\prime}\right)^{\prime}$ $=(a+b)^{\prime \prime}=a+b$.

4.21. $a a=a$.

By 4.7, 4.5, 4.10, $a a=\left(a^{\prime}+a^{\prime}\right)^{\prime}=\left(a^{\prime}\right)^{\prime}=a$.

4.22. $a+U=U$.

By 4.12, 4.4, 4.5, 4.12, $a+U=a+\left(a+a^{\prime}\right)=(a+a)+a^{\prime}=a+a^{\prime}=U$.

4.23. $a z=z$.

By 4.7, 4.13, 4.22, $a z=\left(a^{\prime}+z^{\prime}\right)^{\prime}=\left(a^{\prime}+u\right)^{\prime}=U^{\prime}=z$.

4.24. $a+a b=a$.

By 4.8, $a b+a b^{\prime}=a$. Hence by 4.3, 4.4, 4.5, $a+a b=a b+a=a b+\left(a b+a b^{\prime}\right)$ $=(a b+a b)+a b^{\prime}=a b+a b^{\prime}=a$.

4.25. $a(a+b)=a$.

By 4.7, 4.20,4.10,4.24, 4.10, $a(a+b)=\left[a^{\prime}+(a+b)^{\prime}\right]^{\prime}=\left[a^{\prime}+\left(a^{\prime} b^{\prime}\right)^{\prime \prime}\right]^{\prime}$ $=\left[a^{\prime}+a^{\prime} b^{\prime}\right]^{\prime}=\left[a^{\prime}\right]^{\prime}=a$. 
4.26. If $a^{\prime}+b=U$ and $b^{\prime}+a=U$, then $a=b$.

By 4.15 and 4.3, $a+v^{\prime}=a$. By 4.6, $\left(a^{\prime}+b^{\prime}\right)^{\prime}+\left(a^{\prime}+b\right)^{\prime}=a$. Hence if $a^{\prime}+b=u,\left(a^{\prime}+b^{\prime}\right)^{\prime}=a$. By 4.6, $\left(b^{\prime}+a^{\prime}\right)^{\prime}+\left(b^{\prime}+a\right)^{\prime}=b$. Hence if $b^{\prime}+a=u$, $\left(b^{\prime}+a^{\prime}\right)^{\prime}=b$. Hence by $4.3, a=b$.

4.27. If $a+b=U$ and $a b=z$, then $a^{\prime}=b$.

From $a+b=v$, by $4.10, a^{\prime \prime}+b=U$. From $a b=z$, by $4.7,\left(a^{\prime}+b^{\prime}\right)^{\prime}=z$, whence by $4.10,4.13,4.3, b^{\prime}+a^{\prime}=u$. Hence by $4.26, a^{\prime}=b$.

In the following theorems, parentheses are omitted, in view of the associative laws, 4.4 and 4.19 , and references to these laws, and to the commutative laws, 4.3 and 4.18 , will often be understood.

4.28. $a b c+a b c^{\prime}+a b^{\prime} c+a b^{\prime} c^{\prime}+a^{\prime} b c+a^{\prime} b c^{\prime}+a^{\prime} b^{\prime} c+a^{\prime} b^{\prime} c^{\prime}=U$.

By 4.8, the given sum $=a b+a b^{\prime}+a^{\prime} b+a^{\prime} b^{\prime}=a+a^{\prime}$, and by 4.12, $a+a^{\prime}$ $=v$.

4.29. If $A$ and $B$ are any two distinct terms of the sum in 4.28 , then $A B=z$.

For example, $\left(a b^{\prime} c\right)\left(a^{\prime} b c\right)=\left(a a^{\prime}\right)\left(b^{\prime} c b c\right)=z\left(b^{\prime} c b c\right)=z$ by 4.17 and 4.23.

4.30. $a b+a c=a b c+a b c^{\prime}+a b^{\prime} c$.

By $4.8, a b=a b c+a b c^{\prime}$ and $a c=a b c+a b^{\prime} c$. Hence by $4.5, a b+a c=a b c$ $+a b c^{\prime}+a b^{\prime} c$.

4.31. $[a(b+c)]^{\prime}=a b^{\prime} c^{\prime}+a^{\prime} b c+a^{\prime} b c^{\prime}+a^{\prime} b^{\prime} c+a^{\prime} b^{\prime} c^{\prime}$.

By 4.7, 4.10, $[a(b+c)]^{\prime}=a^{\prime}+(b+c)^{\prime}=a^{\prime}+b^{\prime} c^{\prime}$. But by $4.8 a^{\prime}=a^{\prime} b+a^{\prime} b^{\prime}$ $=a^{\prime} b c+a^{\prime} b c^{\prime}+a^{\prime} b^{\prime} c+a^{\prime} b^{\prime} c^{\prime}$, and by $4.18,4.8, b^{\prime} c^{\prime}=a b^{\prime} c^{\prime}+a^{\prime} b^{\prime} c^{\prime}$. Hence the theorem, by 4.5 .

4.32. $(a b+a c)+[a(b+c)]^{\prime}=U$. (From 4.30, 4.31, by 4.28.)

4.33. $(a b+a c)[a(b+c)]^{\prime}=z$.

Let $A, B, C, D, E, F, G, H$ be the eight terms in 4.28 . By $4.30, a b+a c$ $=A+B+C$, and by 4.31, $[a(b+c)]^{\prime}=D+E+F+G+H$. By 4.29, 4.5, $A D+A E=z+z=z$. Hence by $4.32,4.15,[A(D+E)]^{\prime}=U$, whence by 4.10 , 4.13, $A(D+E)=z$. Similarly, $A(D+E)+A F=z+z=z$, whence $A(D+E+F)$ $=z$. And so on; so that $A(D+E+F+G+H)=z$. By similar reasoning, we find $(A+B+C)(D+E+F+G+H)=z$, which proves the theorem.

4.34. $a(b+c)=a b+a c$. (First form of the distributive law.)

From 4.32 and 4.33 , by $4.27,(a b+a c)^{\prime}=[a(b+c)]^{\prime}$. Hence by 4.14 , $a b+a c=a(b+c)$.

4.35. $a+b c=(a+b)(a+c)$. (Second form of the distributive law.)

By 4.10, 4.7, $a+b c=\left(a^{\prime}\right)^{\prime}+\left(b+c^{\prime}\right)^{\prime}=\left[a^{\prime}\left(b^{\prime}+c^{\prime}\right)\right]^{\prime}$, whence by 4.34, $a+b c=\left[a^{\prime} b^{\prime}+a^{\prime} c^{\prime}\right]^{\prime}$. But also $(a+b)(a+c)=\left[(a+b)^{\prime}+(a+c)^{\prime}\right]^{\prime}=\left[a^{\prime} b^{\prime}\right.$ $\left.+a^{\prime} c^{\prime}\right]^{\prime}$. Hence $a+b c=(a+b)(a+c)$.

These propositions include all the postulates of the first set of 1904 (see $4.1,4.7,4.15,4.16,4.3,4.18,4.35,4.34,4.12,4.17)$; so that any system $(K$, ,$\left.+{ }^{\prime}\right)$ which satisfies Postulates 4.1-4.6 will have all the properties of a 
Boolean algebra, if the logical product, $a \times b$, is defined in terms of + and as in 4.7.

To prepare the way for the definition of the relation $a<b$, we prove the following theorems.

4.36. If $a+b=b$, then $a b=a$; and conversely, if $a b=a$, then $a+b=b$.

If $a+b=b$, then by $4.7,4.20,4.24,4.10$,

$$
a b=\left(a^{\prime}+b^{\prime}\right)^{\prime}=\left[a^{\prime}+(a+b)^{\prime}\right]^{\prime}=\left[a^{\prime}+a^{\prime} b^{\prime}\right]^{\prime}=\left[a^{\prime}\right]^{\prime}=a .
$$

If $a b=a$, then by $4.20,4.7,4.10,4.24$,

$$
a+b=\left(a^{\prime} b^{\prime}\right)^{\prime}=\left[(a b)^{\prime} b^{\prime}\right]^{\prime}=\left[(a b)^{\prime \prime}+b^{\prime \prime}\right]^{\prime \prime}=a b+b=b .
$$

4.37. If $a+b=b$, then $a^{\prime}+b=v$; and conversely, if $a^{\prime}+b=v$, then $a+b=b$.

If $a+b=b$, then by 4.7 and $4.22, a^{\prime}+b=a^{\prime}+(a+b)=\left(a^{\prime}+a\right)+b=u+b$ $=v$. If $a^{\prime}+b=v$, then by $4.20,4.15,4.17,4.34,4.7,4.10,4.15, a+b=\left(a^{\prime} b^{\prime}\right)^{\prime}$ $=\left[a^{\prime} b^{\prime}+z\right]^{\prime}=\left[a^{\prime} b^{\prime}+b b^{\prime}\right]^{\prime}=\left[\left(a^{\prime}+b\right) b^{\prime}\right]^{\prime}=\left(a^{\prime}+b\right)^{\prime}+b=v^{\prime}+b=z+b=b$.

4.38. If $a+b=b$, then $a b^{\prime}=z$; and conversely, if $a b^{\prime}=z$, then $a+b=b$.

If $a+b=b$, then by $4.7,4.12,4.22$,

$$
a b^{\prime}=\left(a^{\prime}+b\right)^{\prime}=\left[a^{\prime}+(a+b)\right]^{\prime}=\left[\left(a^{\prime}+a\right)+b\right]^{\prime}=(u+b)^{\prime}=v^{\prime}=z .
$$

If $a b^{\prime}=z$, then by $4.20,4.10, a^{\prime}+b=\left(a b^{\prime}\right)^{\prime}=z^{\prime}=v$, whence by $4.37, a+b=b$.

4.39. Definition. If $a+b=b$; or if $a b=a$; or if $a^{\prime}+b=v$; or if $a b^{\prime}=z$; then and only then we write $a<b$.

The equivalence of these four forms of the definition follows from 4.36, $4.37,4.38$.

The following theorems are added because of their connection with the fifth and sixth sets, below.

4.40. $a+(b+c)^{\prime}=\left[\left(b^{\prime}+a\right)^{\prime}+\left(c^{\prime}+a\right)^{\prime}\right]^{\prime}$.

By 4.7, 4.10, 4.34, 4.21, 4.24, 4.7, $\left[\left(b^{\prime}+a\right)^{\prime}+\left(c^{\prime}+a\right)^{\prime}\right]^{\prime}=\left(b^{\prime}+a\right)\left(c^{\prime}+a\right)$ $=b^{\prime} c^{\prime}+a b^{\prime}+a c^{\prime}+a a=\left[a+a\left(b^{\prime}+c^{\prime}\right)\right]+b^{\prime} c^{\prime}=[a]+b^{\prime} c^{\prime}=a+(b+c)^{\prime}$.

4.41. $\left(b^{\prime}+c\right)^{\prime}+\left[(a+b)^{\prime}+a+c\right]=v$.

By 4.7, 4.20, 4.16, 4.12, 4.34, 4.35,

$$
\begin{aligned}
\left(b^{\prime}+c\right)^{\prime} & =b c^{\prime}=v b c^{\prime}=\left(a+a^{\prime}\right) b c^{\prime}=a b c^{\prime}+a^{\prime} b c^{\prime} ; \\
(a+b)^{\prime} & =a^{\prime} b^{\prime}=a^{\prime} b^{\prime} u=a^{\prime} b^{\prime}\left(c+c^{\prime}\right)=a^{\prime} b^{\prime} c+a^{\prime} b^{\prime} c^{\prime} \\
a+c & =a u+v c=a\left(b+b^{\prime}\right)+\left(b+b^{\prime}\right) c=a b+a b^{\prime}+b c+b^{\prime} c \\
& =\left(a b c+a b c^{\prime}\right)+\left(a b^{\prime} c+a b^{\prime} c^{\prime}\right)+\left(a b c+a^{\prime} b c\right)+\left(a b^{\prime} c+a^{\prime} b^{\prime} c\right) .
\end{aligned}
$$

Hence the theorem, by 4.28 (with 4.5 ).

4.42. $(a+a)^{\prime}+a=u$. (By 4.5, 4.12.)

4.43. $b^{\prime}+(a+b)=v$. 
By 4.3, 4.4, 4.12, 4.22, $b^{\prime}+(a+b)=a+\left(b+b^{\prime}\right)=a+u=v$.

4.44. $(a+b)^{\prime}+(b+a)=v$. (By 4.3, 4.12.)

\section{INDEPENDENCE PROOFS FOR THE FOURTH SET}

The independence of the postulates of the fourth set is established by the existence of the following examples of systems $\left(K,+,{ }^{\prime}\right)$, each of which violates the like-numbered postulate, and satisfies all the other postulates of the set.

Example 4.1. $K=$ two elements, 0 and 1, with + and ' defined as follows: $0+0=0,1+1=1,0+1=x, 1+0=x ; 0^{\prime}=1,1^{\prime}=0$. Here $x$ is not an element of the class $K$, so that Postulate 4.1 fails. The other postulates are satisfied whenever the indicated combinations are elements of the class.

Example 4.2. $K=$ two elements, 0 and 1, with $a+b$ and $a^{\prime}$ defined as in the accompanying table. Here $x$ is not in the class $K$, so that Postulate 4.2 fails. The other postulates are satisfied whenever the indicated combinations are elements of the class.

$$
\begin{array}{c||cc}
+ & 0 & 1 \\
\hline \hline 0 & 0 & 1 \\
1 & 1 & 1
\end{array} \|=
$$

Example 4.3. In this system, Postulate 4.3 fails, since $5+2=5$ and $2+5$ $=2$. The other postulates will be found to be satisfied.

\begin{tabular}{c||cccccc||c}
+ & 0 & 1 & 2 & 3 & 4 & 5 \\
\hline \hline 0 & 0 & 1 & 2 & 3 & 4 & 5 & \multicolumn{1}{|}{} \\
1 & 1 & 1 & 1 & 1 & 1 & 1 & 1 \\
2 & 2 & 1 & 2 & 1 & 1 & 2 & 0 \\
3 & 3 & 1 & 1 & 3 & 3 & 1 & 3 \\
4 & 4 & 1 & 1 & 4 & 4 & 1 & 5 \\
5 & 5 & 1 & 5 & 1 & 1 & 5 & 4
\end{tabular}

Example 4.4 . Here 4.4 fails, since $(2+1)+3=2+3=1$ while $2+(1+3)$ $=2+0=2$. The other postulates are satisfied.

\begin{tabular}{c||cccc||c}
+ & 0 & 1 & 2 & 3 \\
\hline 0 & $=$ & 1 & 2 & 3 \\
1 & 1 & 1 & 2 & 0 & $=$ \\
2 & 2 & 2 & 2 & 1 & 1 \\
3 & 3 & 0 & 1 & 3 & 0 \\
3 \\
2
\end{tabular}


Example 4.5. Here 4.5 fails since $2+2=1$. The other postulates are satisfied.

\begin{tabular}{|c|c|c|c|c|}
\hline+ & 0 & 1 & 2 & l \\
\hline 0 & 0 & 1 & & 1 \\
\hline 1 & 1 & 1 & & 0 \\
\hline 2 & 2 & 1 & 1 & 2 \\
\hline
\end{tabular}

Example 4.6. Here 4.6 fails since $\left(3^{\prime}+5^{\prime}\right)^{\prime}+\left(3^{\prime}+5\right)^{\prime}=(2+4)^{\prime}+(2+5)^{\prime}$ $=1^{\prime}+1^{\prime}=0+0=0 \neq 3$. The other postulates are found to be satisfied.

\begin{tabular}{c||cccccc||c}
+ & 0 & 1 & 2 & 3 & 4 & 5 \\
\hline \hline 0 & 0 & 1 & 2 & 3 & 4 & 5 \\
1 & 1 & 1 & 1 & 1 & 1 & 1 & \\
2 & 2 & 1 & 2 & 1 & 1 & 1 & \\
3 & 3 & 1 & 1 & 3 & 1 & 1 & 1 \\
4 & 4 & 1 & 1 & 1 & 4 & 1 & \\
5 & 5 & 1 & 1 & 1 & 1 & 5
\end{tabular}

THE FIFTH SET

The following set of independent postulates for Boolean algebra is directly suggested by H. M. Sheffer's postulates of 1913, when these are expressed in terms of $\left(K,+,{ }^{\prime}\right)$.

This fifth set contains one fewer postulate than the fourth set; but the fifth set as a whole seems less simple and natural than the fourth set.

(A trivial preliminary postulate 5.0 demanding that the class contain at least two elements is assumed without further mention, and in Postulates 5.3-5.5 it is assumed that the indicated combinations are elements of $K$. Also, Postulates A, B, C, D are assumed without further mention.)

Postulate 5.1. If $a$ and $b$ are in the class $K$, then $a+b$ is in the class $K$.

Postulate 5.2. If $a$ is in the class $K$, then $a^{\prime}$ is in the class $K$.

Postulate 5.3. $\left(a^{\prime}\right)^{\prime}=a$.

Postulate 5.4. $a+\left(b+b^{\prime}\right)^{\prime}=a$.

Postulate 5.5. $a+(b+c)^{\prime}=\left[\left(b^{\prime}+a\right)^{\prime}+\left(c^{\prime}+a\right)^{\prime}\right]^{\prime}$.

The consistency of these five postulates is shown by any example of a Boolean algebra $\left(K,+,{ }^{\prime}\right)$, like the regions within a square, with + and ' defined in the usual way.

The equivalence between the fifth set and the earlier sets is established as follows.

5.6. $a+a=a$. 
By 5.5 and $5.3, b^{\prime}+\left(b+b^{\prime}\right)^{\prime}=\left[\left(b^{\prime}+b^{\prime}\right)^{\prime}+\left(b+b^{\prime}\right)^{\prime}\right]^{\prime}$, whence, applying 5.4 to each side, $b^{\prime}=\left[\left(b^{\prime}+b^{\prime}\right)^{\prime}\right]^{\prime}$. Hence by $5.3, b^{\prime}=b^{\prime}+b^{\prime}$. Hence $a^{\prime \prime}=a^{\prime \prime}$ $+a^{\prime \prime}$, whence by $5.3, a=a+a$.

5.7. $a+b=b+a$.

By 5.3, 5.6, 5.5, 5.6, 5.3,

$$
\begin{aligned}
a+b & =a+\left(b^{\prime}\right)^{\prime}=a+\left(b^{\prime}+b^{\prime}\right)^{\prime}=\left[\left(b^{\prime \prime}+a\right)^{\prime}+\left(b^{\prime \prime}+a\right)^{\prime}\right]^{\prime} \\
& =\left[\left(b^{\prime \prime}+a\right)^{\prime}\right]^{\prime}=b+a .
\end{aligned}
$$

5.8. $\left(a^{\prime}+b^{\prime}\right)^{\prime}+\left(a^{\prime}+b\right)^{\prime}=a$.

By 5.4, 5.5, 5.3, 5.7,

$$
\begin{aligned}
a^{\prime} & =a^{\prime}+\left(b+b^{\prime}\right)^{\prime}=\left[\left(b^{\prime}+a^{\prime}\right)^{\prime}+\left(b^{\prime \prime}+a^{\prime}\right)^{\prime}\right]^{\prime}=\left[\left(b^{\prime}+a^{\prime}\right)^{\prime}+\left(b+a^{\prime}\right)^{\prime}\right]^{\prime} \\
& =\left[\left(a^{\prime}+b^{\prime}\right)^{\prime}+\left(a^{\prime}+b\right)^{\prime}\right]^{\prime} .
\end{aligned}
$$

Hence by $5.3, a=\left(a^{\prime}+b^{\prime}\right)^{\prime}+\left(a^{\prime}+b\right)^{\prime}$.

The following theorems lead up to the associative law, 5.27.

5.9. $a+a^{\prime}=b+b^{\prime}$.

By $5.4,5.7,5.4$,

$\left(a+a^{\prime}\right)^{\prime}=\left(a+a^{\prime}\right)^{\prime}+\left(b+b^{\prime}\right)^{\prime}=\left(b+b^{\prime}\right)^{\prime}+\left(a+a^{\prime}\right)^{\prime}=\left(b+b^{\prime}\right)^{\prime}$.

Hence by $5.3, a+a^{\prime}=b+b^{\prime}$.

5.10. Definition. $v=a+a^{\prime}=$ the "universe" element of the system.

This element $U$ exists, by 5.1 and 5.2, and is unique by 5.9. Moreover, by 5.7, $u=a^{\prime}+a$.

5.11. Definition. $z=\left(a+a^{\prime}\right)^{\prime}=$ the "zero" element of the system.

This element $z$ exists, by 5.1 and 5.2 , and is unique by 5.9. Obviously, $z=U^{\prime}$, where $U=$ the universe element of 5.10 ; and by $5.3, z^{\prime}=U$.

5.12. $a+z=a$.

By 5.11 and 5.4, $a+z=a+\left(a+a^{\prime}\right)^{\prime}=a$.

5.13. $a+U=U$.

By 5.10, 5.12, 5.7, 5.5, 5.3, 5.11, 5.12, 5.3, $\quad=a+a^{\prime}=a+(a+z)^{\prime}=$ $a+\left(u^{\prime}+a\right)^{\prime}=\left[\left(v^{\prime \prime}+a\right)^{\prime}+\left(a^{\prime}+a\right)^{\prime}\right]^{\prime}=\left[(u+a)^{\prime}+v^{\prime}\right]^{\prime}=\left[(a+v)^{\prime}+z\right]^{\prime}=(a+v)^{\prime \prime}$ $=a+U$.

5.14. Definition. $a b=\left(a^{\prime}+b^{\prime}\right)^{\prime}$.

By 5.1 and 5.2, if $a$ and $b$ are in the class $K$, then $a b$ is in the class $K$.

5.15. $a a=a$.

By 5.14, 5.6, 5.3, $a a=\left(a^{\prime}+a^{\prime}\right)^{\prime}=\left(a^{\prime}\right)^{\prime}=a$.

5.16. $a b=b a$. (By 5.14 and 5.7.)

5.17. $a a^{\prime}=z$.

By 5.14, 5.10, 5.11, $a a^{\prime}=\left(a^{\prime}+a\right)^{\prime}=U^{\prime}=z$.

5.18. $a z=z$. 
By 5.14, 5.11, 5.13, 5.11, $a z=\left(a^{\prime}+z^{\prime}\right)^{\prime}=\left(a^{\prime}+v\right)^{\prime}=U^{\prime}=z$.

5.19. $a U=a$.

By 5.14, 5.11, 5.12, 5.3, $a v=\left(a^{\prime}+U^{\prime}\right)^{\prime}=\left(a^{\prime}+z\right)^{\prime}=\left(a^{\prime}\right)^{\prime}=a$.

5.20. $a+b=\left(a^{\prime} b^{\prime}\right)^{\prime}$. (By 5.14 and 5.3.)

5.21. $a+b c=(a+b)(a+c)$.

By 5.14, 5.5 and 5.3, 5.14, 5.7, $a+b c=a+\left(b^{\prime}+c^{\prime}\right)^{\prime}=\left[(b+a)^{\prime}+(c+a)^{\prime}\right]^{\prime}$ $=(b+a)(c+a)=(a+b)(a+c)$.

5.22. $a(b+c)=a b+a c$.

By 5.14, 5.14 and 5.3, 5.21, 5.14 and 5.3, 5.14, we have $a(b+c)=$ $\left[a^{\prime}+(b+c)^{\prime}\right]^{\prime}=\left[a^{\prime}+b^{\prime} c^{\prime}\right]^{\prime}=\left[\left(a^{\prime}+b^{\prime}\right)\left(a^{\prime}+c^{\prime}\right)\right]^{\prime}=\left(a^{\prime}+b^{\prime}\right)^{\prime}+\left(a^{\prime}+c^{\prime}\right)^{\prime}=a b+a c$.

5.23. $a+a b=a$.

By 5.19, 5.22, 5.13 and 5.7, 5.19, $a+a b=a U+a b=a(U+b)=a U=a$.

5.24. $a(a+b)=a$.

By 5.22, 5.15, 5.23, $a(a+b)=a a+a b=a+a b=a$.

5.25. If $a^{\prime}+b=v$ and $b^{\prime}+a=v$, then $a=b$.

By 5.11 and 5.12, $a+U^{\prime}=a$. By 5.8, $\left(a^{\prime}+b^{\prime}\right)^{\prime}+\left(a^{\prime}+b\right)^{\prime}=a$. Hence if $a^{\prime}+b=v$, then by $5.12, a^{\prime}+b^{\prime}=a$. By $5.8,\left(b^{\prime}+a^{\prime}\right)^{\prime}+\left(b^{\prime}+a\right)^{\prime}=b$. Hence if $b^{\prime}+a=u$, then by $5.12, b^{\prime}+a^{\prime}=b$. Hence by $5.7, a=b$.

5.26. If $a+b=u$ and $a b=z$, then $a^{\prime}=b$.

From $a+b=v$, by $5.3, a^{\prime \prime}+b=U$. From $a b=z$, by 5.14, $\left(a^{\prime}+b^{\prime}\right)^{\prime}=z$, whence by $5.3,5.7,5.11, b^{\prime}+a^{\prime}=u$. Hence by $5.25, a^{\prime}=b$.

5.27. $(a+b)+c=a+(b+c)$.

We prove first the following lemmas.

LEMMA (1). If $x=(a+b)+c$, then $a x=a, b x=b$, and $c x=c$.

For, by $5.7,5.22,5.24,5.23$,

$$
a x=a[(a+b)+c]=a(a+b)+a c=a+a c=a,
$$

whence similarly, $b x=b$; and by 5.7, 5.22, 5.15, 5.19, 5.22, 5.13, 5.19, $c x=c[(a+b)+c]=c(a+b)+c c=c(a+b)+c=c(a+b)+c U=c[(a+b)+v]=c U$ $=c$.

Lemma (2). If $x=(a+b)+c$, then $x^{\prime} a=z, x^{\prime} b=z$, and $x^{\prime} c=z$.

For, by 5.19 and 5.7, 5.10, 5.21, Lemma (1), 5.10, $x+a^{\prime}=v\left(a^{\prime}+x\right)$ $=\left(a^{\prime}+a\right)\left(a^{\prime}+x\right)=a^{\prime}+a x=a^{\prime}+a=U$, whence by 5.14, 5.11, $x^{\prime} a=\left(x+a^{\prime}\right)^{\prime}$ $=U^{\prime}=z$. Similarly, $x^{\prime} b=z$ and $x^{\prime} c=z$.

LemMa (3). If $y=a+(b+c)$, then $y+a^{\prime}=v, y+b^{\prime}=v$, and $y+c^{\prime}=v$.

For, by Lemma (2), with 5.7, $y^{\prime} a=z, y^{\prime} b=z, y^{\prime} c=z$, whence, by 5.20, 5.11, $y+a^{\prime}=\left(y^{\prime} a\right)^{\prime}=z^{\prime}=U$. Similarly $y+b^{\prime}=U$ and $y+c^{\prime}=v$. 
The proof of the main theorem then proceeds as follows. By 5.22, 5.22, 5.6, $x^{\prime} y=x^{\prime}[a+(b+c)]=x^{\prime} a+x^{\prime}(b+c)=x^{\prime} a+\left(x^{\prime} b+x^{\prime} c\right)=z+(z+z)=z+z$ $=z ;$ and by $5.7,5.20$ and $5.3,5.21,5.21,5.19$,

$$
\begin{aligned}
x^{\prime}+y & =y+[c+(b+a)]^{\prime}=y+\left[c^{\prime}(b+a)^{\prime}\right]=y+\left[c^{\prime}\left(b^{\prime} a^{\prime}\right)\right] \\
& =\left(y+c^{\prime}\right)\left[y+\left(b^{\prime} a^{\prime}\right)\right]=\left(y+c^{\prime}\right)\left[\left(y+b^{\prime}\right)\left(y+a^{\prime}\right)\right]=v(v U)=v U=v .
\end{aligned}
$$

Hence by 5.26, $x=y$; that is, $(a+b)+c=a+(b+c)$.

We can now establish the equivalence of the fifth set and the fourth set, as follows:

Theorems 5.1, 5.2, 5.6, 5.7, 5.8, and 5.27 show that all the postulates of the fourth set are deducible from Postulates 5.1-5.5; and conversely all the postulates of the fifth set are readily deducible from Postulates 4.1-4.6.

Incidentally, Theorems 5.1, 5.14, 5.12, 5.19, 5.7, 5.16, 5.21, 5.22, 5.10 and 5.17 show directly that all the postulates of the "first set" are deducible from Postulates 5.1-5.5 (when the product $a b$ is defined as in 5.14); and conversely, all the postulates of the fifth set are readily deducible from Postulates Ia-VI (when $a^{\prime}$ is defined in terms of + and $X$ as in V); so that the equivalence between the fifth set and the "first set" is established directly, without reference to the fourth set.

To show that the fifth set of postulates is equivalent to Sheffer's set of 1913, which occupies so important a position in the revised edition of the Principia Mathematica (volume 1, 1925), we need the definition of Sheffer's "stroke" function, namely:

5.28. Definition. $a \mid b=(a+b)^{\prime}=$ the "reject" of $a$ and $b$ (pronounced $a$ per $b)$.

On the basis of this definition we deduce Sheffer's postulates from Postulates 5.1-5.5 as follows:

(1) There are at least two distinct $K$-elements.

(2) Whenever $a$ and $b$ are $K$-elements, $a \mid b$ is a $K$-element. (By 5.1, 5.2.)

Definition. $a^{\prime}=a \mid a$. (By 5.6.)

(3) $\left(a^{\prime}\right)^{\prime}=a$. (By 5.3.)

(4) $a \mid\left(b \mid b^{\prime}\right)=a^{\prime}$. (By 5.4 and 5.3.)

(5) $[a \mid(b \mid c)]^{\prime}=\left(b^{\prime} \mid a\right) \mid\left(c^{\prime} \mid a\right)$. (By 5.5 and 5.3.)*

* In a paper published in 1916, B. A. Bernstein showed that Sheffer's postulates (3), (4), and (5) may be replaced by two postulates, $P_{3}$ and $P_{4}$ :

P. $(b \mid a) \mid\left(b^{\prime} \mid a\right)=a$.

P. $a^{\prime} \mid\left(b^{\prime} \mid c\right)=\left[\left(b \mid a^{\prime}\right) \mid\left(c^{\prime} \mid a^{\prime}\right)\right]^{\prime}$.

This change does not lead to any corresponding reduction in Postulates 5.1-5.5. 
INDEPENDENCE PROOFS FOR THE FIFTH SET

The independence of the postulates of the fifth set is established by the existence of the following examples of systems $\left(K,+,{ }^{\prime}\right)$, each of which violates the like-numbered postulate, and satisfies all the other postulates of the set.

Example 5.1. $K=$ two elements, 0,1 , with $a+b$ and $a^{\prime}$ defined as in the accompanying table, where $x$ is any object not an element of the class $K$.

\begin{tabular}{|c|c|c|}
\hline+1 & \begin{tabular}{|ll}
$\mid$ & 0
\end{tabular} & \\
\hline$\overline{0}$ & & 1 \\
\hline 1 & $\begin{array}{ll}1 & x\end{array}$ & 110 \\
\hline
\end{tabular}

Example 5.2. $K=$ two elements, 0,1 , with $a+b$ and $a^{\prime}$ given by the table ( $x$ being any object not an element of the class $K$ ).

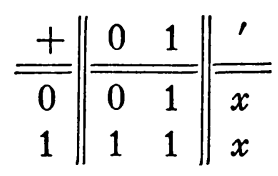

Example 5.3. $K=$ six elements, with $a+b$ and $a^{\prime}$ given by the table.

\begin{tabular}{l||cccccc||c}
+ & 0 & 1 & 2 & 3 & 4 & 5 & \multicolumn{1}{|c}{} \\
\hline \hline 0 & 0 & 1 & 4 & 5 & 2 & 3 & 1 \\
1 & 1 & 1 & 1 & 1 & 1 & 1 & \\
2 & 2 & 1 & 0 & 1 & 2 & 1 & 0 \\
3 & 3 & 1 & 1 & 0 & 1 & 3 & 3 \\
4 & 4 & 1 & 4 & 1 & 0 & 1 & 4 \\
5 & 5 & 1 & 1 & 5 & 1 & 0 & 2
\end{tabular}

Here Postulate 5.3 fails, since $\left(2^{\prime}\right)^{\prime}=3^{\prime}=4$. All the other postulates of the fifth set are found to be satisfied.

It is interesting to note that while the commutative law, $a+b=b+a$, does not hold in this example, it is always true that $a+b=(b+a)^{\prime \prime}$.

Example 5.4. $K=$ three elements, $0,1,2$, with $a+b$ and $a^{\prime}$ given by the table.

\begin{tabular}{c||ccc||c}
+ & 0 & 1 & 2 \\
\hline 0 & 0 & 1 & 2 \\
1 & 1 & 1 & 1 \\
2 & 2 & 1 & 2
\end{tabular}|| $\begin{gathered}\prime \\
1 \\
0 \\
2\end{gathered}$

Here Postulate 5.4 fails, since $0+\left(2+2^{\prime}\right)^{\prime}=2$. 
Example 5.5. $K=$ three elements, $0,1,2$, with $a+b$ and $a^{\prime}$ given by the table.

\begin{tabular}{|c|c|c|c|c|}
\hline+ & 0 & 1 & 2 & \\
\hline 0 & & 2 & & \\
\hline 1 & & 1 & & \\
\hline 2 & 2 & 0 & & \\
\hline
\end{tabular}

To show that Postulate 5.5 fails, take $a=1, b=1, c=2$.

\section{THE SIXTH SET}

The following set of postulates for Boolean algebra in terms of $\left(K,+,{ }^{\prime}\right)$ is suggested by B. A. Bernstein's version of the primitive propositions of the Principia. The only modifications are as follows: (a) his proposition 1.5 is omitted because it can be proved as a theorem; (b) his notation " $=1$ " is here replaced by the notation "is in a subclass $T$ ", which corresponds more nearly to the Frege assertion sign, 1 ; and (c) our Postulate 6.8 is an additional postulate, not included among the primitive propositions of the Principia.

A trivial preliminary postulate, 6.0 , demanding that the class $K$ contain at least two distinct elements, is assumed without further mention; and in Postulates 6.4-6.8 the indicated combinations are assumed to be elements of $K$. Also, Postulates A, B, C,D are assumed without further mention. The numbers in brackets indicate the corresponding postulates in the BernsteinPrincipia list.

Postulate 6.1. [1.71.] If $a$ and $b$ are in the class $K$, then $a+b$ is in the class $K$.

Postulate 6.2. [1.7.] If $a$ is in the class $K$, then $a^{\prime}$ is in the class $K$.

There exists in the class $K$ a subclass $T$ having the following five properties:

Postulate 6.3. [1.1.] If $a$ is in $T$ and $a^{\prime}+b$ is in $T$, then $b$ is in $T$.

Postulate 6.4. [1.2.] If $a$ is in $K$, then $(a+a)^{\prime}+a$ is in $T$.

Postulate 6.5. [1.3.] If $a$ and $b$ are in $K$, then $b^{\prime}+(a+b)$ is in $T$.

Postulate 6.6. [1.4.] If $a$ and $b$ are in $K$, then $(a+b)^{\prime}+(b+a)$ is in $T$.

Postulate 6.7. [1.6.] If $a, b, c$ are in $K$, then $\left(b^{\prime}+c\right)^{\prime}+\left[(a+b)^{\prime}+(a+c)\right]$ is in $T$.

Postulate 6.8. If $T$ is a subclass having the five properties just mentioned, then we have: If $a^{\prime}+b$ is in $T$, and $b^{\prime}+a$ is in $T$, then $a=b$.

The consistency of these postulates is established by the existence of any Boolean algebra $\left(K,+,^{\prime}\right)$, with the subclass $T$ taken as the class containing the single element $v$. 
The equivalence of the sixth set and the fourth set is established as follows.

In the first place, all the postulates of the sixth set are readily deducible from the fourth set; the single element $v$ (see 4.12) constitutes the required subclass $T$.

We proceed to show, conversely, that all the postulates of the fourth set can be derived as theorems from the sixth set.

6.9. If $a^{\prime}+b$ is in $T$ and $b^{\prime}+c$ is in $T$, then $a^{\prime}+c$ is in $T$.

By $6.7,\left(b^{\prime}+c\right)^{\prime}+\left[\left(a^{\prime}+b\right)^{\prime}+\left(a^{\prime}+c\right)\right]$ is in $T$. But by hypothesis, $b^{\prime}+c$ is in $T$. Hence by $6.3,\left(a^{\prime}+b\right)^{\prime}+\left(a^{\prime}+c\right)$ is in $T$. But by hypothesis, $a^{\prime}+b$ is in $T$. Hence by $6.3, a^{\prime}+c$ is in $T$.

Note. This theorem 6.9 corresponds to the "syllogism" in the theory of deduction, while 6.3 corresponds to the "rule of inference."

By the aid of this theorem we can establish at once the redundancy of proposition 1.5 in the Bernstein-Principia list. This theorem 1.5 will serve as a lemma in the proof of the associative law (6.12).

6.10. [1.5.] $[a+(b+c)]^{\prime}+[b+(a+c)]$ is in $T$.

(The following proof is adapted from a proof given, in another notation, by P. Bernays in 1926. It does not involve Postulate 6.8.)

By $6.5, c^{\prime}+(a+c)$ is in $T$.

By 6.7, $\left[c^{\prime}+(a+c)\right]^{\prime}+\left\{(b+c)^{\prime}+[b+(a+c)]\right\}$ is in $T$.

Hence by $6.3,(b+c)^{\prime}+[b+(a+c)]$ is in $T$.

By 6.7, $\left\{(b+c)^{\prime}+[b+(a+c)]\right\}^{\prime}+\left([a+(b+c)]^{\prime}+\{a+[b+(a+c)]\}\right)$ is in $T$.

Hence by $6.3,[a+(b+c)]^{\prime}+\{a+[b+(a+c)]\}$ is in $T$.

By 6.6, $\{a+[b+(a+c)]\}^{\prime}+\{[b+(a+c)]+a\}$ is in $T$.

Hence by 6.9 ,

$$
[a+(b+c)]^{\prime}+\{[b+(a+c)]+a\} \text { is in } T .
$$

By $6.5, a^{\prime}+(c+a)$ is in $T$.

By 6.6, $(c+a)^{\prime}+(a+c)$ is in $T$.

Hence by $6.9, a^{\prime}+(a+c)$ is in $T$.

By 6.5, $(a+c)^{\prime}+[b+(a+c)]$ is in $T$.

Hence by $6.9, a^{\prime}+[b+(a+c)]$ is in $T$.

By 6.7, $\left\{a^{\prime}+[b+(a+c)]\right\}^{\prime}+\left(\{[b+(a+c)]+a\}^{\prime}+\{[b+(a+c)]+[b+(a\right.$ $+c)]\})$ is in $T$.

Hence by $6.3,\{[b+(a+c)]+a\}^{\prime}+\{[b+(a+c)]+[b+(a+c)]\}$ is in $T$.

By 6.4, $\{[b+(a+c)]+[b+(a+c)]\}^{\prime}+[b+(a+c)]$ is in $T$.

Hence by 6.9 ,

$$
\{[b+(a+c)]+a\}^{\prime}+[b+(a+c)] \text { is in } T .
$$


From (1) and (2), by 6.9, $[a+(b+c)]^{\prime}+[b+(a+c)]$ is in $T$.

The next three theorems correspond to Postulates 4.3, 4.4, 4.5 of the fourth set.

6.11. $a+b=b+a$.

By 6.6, $(a+b)^{\prime}+(b+a)$ is in $T$. Again, by $6.6,(b+a)^{\prime}+(a+b)$ is in $T$. Hence by $6.8, a+b=b+a$.

6.12. $(a+b)+c=a+(b+c)$.

By 6.10, $[a+(c+b)]^{\prime}+[c+(a+b)]$ is in $T$. Hence by 6.11 ,

$$
[a+(b+c)]^{\prime}+[(a+b)+c] \text { is in } T .
$$

From (1), $[c+(b+a)]^{\prime}+[(c+b)+a]$ is in $T$. Hence by 6.11 ,

$$
[(a+b)+c]^{\prime}+[a+(b+c)] \text { is in } T \text {. }
$$

From (1) and (2), by $6.8, a+(b+c)=(a+b)+c$.

6.13. $a+a=a$.

By 6.4, $(a+a)^{\prime}+a$ is in $T$. By $6.5, a^{\prime}+(a+a)$ is in $T$. Hence by $6.8, a+a$ $=a$.

The following theorems establish the existence and properties of the universe element $v$.

6.14. If $a$ is in $K$, then $a+a^{\prime}$ is in $T$.

By $6.7\left[(a+a)^{\prime}+a\right]^{\prime}+\left\{\left[a^{\prime}+(a+a)\right]^{\prime}+\left(a^{\prime}+a\right)\right\}$ is in $T$. But by 6.4, $(a+a)^{\prime}+a$ is in $T$. Hence by $6.3,\left[a^{\prime}+(a+a)\right]^{\prime}+\left(a^{\prime}+a\right)$ is in $T$. But by $6.5, a^{\prime}+(a+a)$ is in $T$. Hence by $6.3, a^{\prime}+a$ is in $T$. Hence by $6.11, a+a^{\prime}$ is in $T$.

6.15. $a+a^{\prime}=b+b^{\prime}$.

By 6.7, $\left[(a+b)^{\prime}+(b+a)\right]^{\prime}+\left\{\left[b^{\prime}+(a+b)\right]^{\prime}+\left[b^{\prime}+(b+a)\right]\right\}$ is in T. But by $6.6,(a+b)^{\prime}+(b+a)$ is in $T$. Hence by $6.3,\left[b^{\prime}+(a+b)\right]^{\prime}+\left[b^{\prime}+(b+a)\right]$ is in $T$. But by $6.5, b^{\prime}+(a+b)$ is in $T$. Hence by 6.3 ,

$$
b^{\prime}+(b+a) \text { is in } T .
$$

From (1), $\left(a+a^{\prime}\right)^{\prime}+\left[\left(a+a^{\prime}\right)+\left(b+b^{\prime}\right)^{\prime}\right]$ is in $T$. But by 6.14, $a+a^{\prime}$ is in $T$. Hence by 6.3 ,

$$
\left(a+a^{\prime}\right)+\left(b+b^{\prime}\right)^{\prime} \text { is in } T .
$$

Now by 6.6, $\left[\left(a+a^{\prime}\right)+\left(b+b^{\prime}\right)^{\prime}\right]^{\prime}+\left[\left(b+b^{\prime}\right)^{\prime}+\left(a+a^{\prime}\right)\right]$ is in $T$. Hence by (2) and 6.3,

$$
\left(b+b^{\prime}\right)^{\prime}+\left(a+a^{\prime}\right) \text { is in } T .
$$

From (3),

$$
\left(a+a^{\prime}\right)^{\prime}+\left(b+b^{\prime}\right) \text { is in } T .
$$


From (4) and (3), by $6.8, b+b^{\prime}=a+a^{\prime}$.

This theorem justifies the following definition.

6.16. Definition. $U=a+a^{\prime}=$ the "universe" element of the system.

This element $U$ exists, by 6.1 and 6.2 , and is unique, by 6.15 . Also, by $6.11, U=a^{\prime}+a$; and obviously, by $6.14, v$ is in $T$.

6.17. If $a$ is in $T$, then $a=U$.

By 6.5,

$$
a^{\prime}+\left(a^{\prime}+a\right) \text { is in } T .
$$

Again, by $6.5, a^{\prime}+\left[\left(a^{\prime}+a\right)^{\prime}+a\right]$ is in $T$. But by hypothesis, $a$ is in $T$. Hence by 6.3 ,

$$
\left(a^{\prime}+a\right)^{\prime}+a \text { is in } T \text {. }
$$

From (1) and (2), by 6.8, $a=a^{\prime}+a$. Hence by 6.16, $a=v$.

This theorem shows that the subclass $T$ consists of a single element of the class $K$, namely, the element $v$ defined in 6.16 .

6.18. $a+v=v$ (whence, by 6.11, $v+a=v$ ).

By $6.5, v^{\prime}+(a+v)$ is in $T$. But by $6.16, v$ is in $T$. Hence by $6.3, a+v$ is in $T$. Hence by $6.17, a+U=U$.

6.19. $v^{\prime}+a=a$ (whence, by 6.11, $\left.a+v^{\prime} \doteq a\right)$.

By 6.7, $\left(u^{\prime}+a\right)^{\prime}+\left[(a+v)^{\prime}+(a+a)\right]$ is in $T$. Hence by 6.18 and 6.13, $\left(v^{\prime}+a\right)^{\prime}+\left(v^{\prime}+a\right)$ is in $T$. Hence by 6.11 and $6.12, v^{\prime}+\left[\left(v^{\prime}+a\right)^{\prime}+a\right]$ is in $T$. Hence by $6.3,\left(v^{\prime}+a\right)^{\prime}+a$ is in $T$. But by $6.5, a^{\prime}+\left(U^{\prime}+a\right)$ is in $T$. Hence by 6.8, $v^{\prime}+a=a$.

6.20. $U^{\prime} \neq u$.

Suppose $U^{\prime}=U$. Then by 6.19 we would have $a+U=a$. But by 6.18 , $a+v=v$. Hence we would have $a=v$; that is, every element $a$ would be equal to the element $U$, so that the class $K$ would contain only a single element. This trivial case is excluded by Postulate 6.0.

6.21. $a^{\prime}=a$ is always false.

Suppose there existed an element $c$ such that $c^{\prime}=c$. Then by 6.13 and 6.16, $c=c+c=c+c^{\prime}=u$. Hence $c^{\prime}=u^{\prime}$. Hence, if $c^{\prime}=c$ we would have $U^{\prime}=u$, which by 6.20 is impossible.

6.22. If $a$ is in $T$, then $a^{\prime}$ is not in $T$.

Since $a$ is in $T$, by $6.17, a=U$, whence by $6.2, a^{\prime}=U^{\prime}$. If $a^{\prime}$ also were in $T$, then, by $6.17, a^{\prime}=U$, whence $U^{\prime}=U$, which by 6.20 is impossible.

6.23. $a^{\prime \prime}=a$.

By 6.7, $\left(a^{\prime \prime}+a^{\prime \prime \prime}\right)^{\prime}+\left[\left(a+a^{\prime}\right)^{\prime}+\left(a+a^{\prime \prime \prime}\right)\right]$ is in $T$. But by 6.14, $a^{\prime \prime}+a^{\prime \prime \prime}$ is in $T$. Hence by $6.3,\left(a+a^{\prime}\right)^{\prime}+\left(a+a^{\prime \prime \prime}\right)$ is in $T$. But by $6.14, a+a^{\prime}$ is in $T$. 
Hence by $6.3, a+a^{\prime \prime \prime}$ is in $T$, whence by 6.11 ,

$$
a^{\prime \prime \prime}+a \text { is in } T \text {. }
$$

Again, by 6.14,

$$
a^{\prime}+a^{\prime \prime} \text { is in } T .
$$

From (1) and (2), by 6.8, $a^{\prime \prime}=a$.

6.24. Definition. $a b=\left(a^{\prime}+b^{\prime}\right)^{\prime}$.

6.25. $a b=b a$. (By 6.24 and 6.11.)

6.26. $(a b) c=a(b c)$. (By 6.24, 6.23, 6.12.)

6.27. $a a=a$. (By $6.24,6.23,6.13$.)

6.28. $a v=a$.

By 6.24, 6.11, 6.19, 6.23, $a U=\left(a^{\prime}+U^{\prime}\right)^{\prime}=\left(u^{\prime}+a^{\prime}\right)^{\prime}=\left(a^{\prime}\right)^{\prime}=a$.

6.29. Definition. $z=U^{\prime}=$ the "zero" element of the system.

6.30. $a+z=a$. (By 6.29 and 6.19.)

6.31. $a z=z$.

By 6.24, 6.29, 6.23, 6.18, $a z=\left(a^{\prime}+z^{\prime}\right)^{\prime}=\left(a^{\prime}+v\right)^{\prime}=v^{\prime}=z$.

6.32. $a a^{\prime}=z$.

By 6.24, 6.16, 6.29, $a a^{\prime}=\left(a^{\prime}+a^{\prime \prime}\right)^{\prime}=u^{\prime}=z$.

6.33. $(a b)^{\prime}=a^{\prime}+b^{\prime}$. (By 6.24, 6.23.)

6.34. $(a+b)^{\prime}=a^{\prime} b^{\prime}$. (By 6.24, 6.23.)

In the following proofs, tacit use will be made of 6.17 .

6.35. If $a^{\prime}+b=v$, then $a b=a$.

By 6.7, $\left[b^{\prime}+\left(a^{\prime}+a b\right)\right]^{\prime}+\left\{\left(a^{\prime}+b\right)^{\prime}+\left[a^{\prime}+\left(a^{\prime}+a b\right)\right]\right\}=v$. But by 6.11, 6.12, 6.24, 6.16, $b^{\prime}+\left(a^{\prime}+a b\right)=\left(a^{\prime}+b^{\prime}\right)+a b=\left(a^{\prime}+b^{\prime}\right)+\left(a^{\prime}+b^{\prime}\right)^{\prime}=v$. Hence by $6.3,\left(a^{\prime}+b\right)^{\prime}+\left[a^{\prime}+\left(a^{\prime}+a b\right)\right]=v$. But by hypothesis, $a^{\prime}+b=v$. Hence by $6.3, a^{\prime}+\left(a^{\prime}+a b\right)=v$, whence by 6.12 and 6.13 ,

$$
a^{\prime}+a b=U .
$$

Again, by 6.33, 6.11, 6.12, 6.16, $(a b)^{\prime}+a=\left(a^{\prime}+b^{\prime}\right)+a=b^{\prime}+\left(a+a^{\prime}\right)=b^{\prime}+v$, whence by 6.18 ,

$$
(a b)^{\prime}+a=v .
$$

From (1) and (2), by $6.8, a=a b$.

6.36. If $a b^{\prime}=z$, then $a+b=b$.

From $a b^{\prime}=z$, by $6.33,6.25,6.29, b^{\prime \prime}+a^{\prime}=\left(b^{\prime} a\right)^{\prime}=\left(a b^{\prime}\right)^{\prime}=z^{\prime}=v$. Hence by $6.35, b^{\prime} a^{\prime}=b^{\prime}$, whence by $6.34,(b+a)^{\prime}=b^{\prime}$. Hence by $6.11,6.23, a+b=b$.

6.37. If $a b^{\prime}=z$, then $a b=a$.

From $a b^{\prime}=z$, by $6.24,6.23,6.29, a^{\prime}+b=v$. Hence by $6.35, a b=a$.

6.38. If $a b=a$, then $a+b=b$. 
From $a b=a$, by $6.33, a^{\prime}=a^{\prime}+b^{\prime}$. Hence by $6.12,6.13,6.16,6.18,\left(a^{\prime}\right)+b$ $=\left(a^{\prime}+b^{\prime}\right)+b=a^{\prime}+\left(b+b^{\prime}\right)=a^{\prime}+u=v$, whence by $6.25,6.23, b^{\prime \prime}+a^{\prime}=v$. Hence by $6.35, b^{\prime} a^{\prime}=b^{\prime}$, whence by $6.34,(b+a)^{\prime}=b^{\prime}$. Hence by $6.25,6.23$, $a+b=b$.

6.39. $a\left(a b+a b^{\prime}\right)^{\prime}=z$.

Let $x=a\left(a b+a b^{\prime}\right)^{\prime}$. Then by $6.26,6.25,6.32,6.31$,

(1) $x\left(a b+a b^{\prime}\right)=\left[a\left(a b+a b^{\prime}\right)^{\prime}\right]\left(a b+a b^{\prime}\right)=a\left[\left(a b+a b^{\prime}\right)^{\prime}\left(a b+a b^{\prime}\right)\right]=a z=z$;

$$
x a^{\prime}=\left[a\left(a b+a b^{\prime}\right)^{\prime}\right] a^{\prime}=\left(a b+a b^{\prime}\right)^{\prime}\left(a a^{\prime}\right)=\left(a b+a b^{\prime}\right) z=z .
$$

From (1), by $6.25,6.23,\left(a b+a b^{\prime}\right) x^{\prime \prime}=z$, whence by $6.36, a b+a b^{\prime}+x^{\prime}=x^{\prime}$. Hence by $6.11,6.12,6.13, a b+x^{\prime}=a b+\left(a b+a b^{\prime}+x^{\prime}\right)=a b+a b^{\prime}+x^{\prime}=x^{\prime}$, whence by $6.24,6.23$,

$$
(a b)^{\prime} x=x ;
$$

also, $a b^{\prime}+x^{\prime}=a b^{\prime}+\left(a b+a b^{\prime}+x^{\prime}\right)=a b+a b^{\prime}+x^{\prime}=x^{\prime}$, whence by 6.24, 6.23,

$$
\left(a b^{\prime}\right)^{\prime} x=x .
$$

From (4), by $6.26,6.25,6.32,6.31$,

$$
(x a) b^{\prime}=x\left(a b^{\prime}\right)=\left[\left(a b^{\prime}\right)^{\prime} x\right]\left(a b^{\prime}\right)=x\left[\left(a b^{\prime}\right)\left(a b^{\prime}\right)^{\prime}\right]=x z=z,
$$

whence by $6.37,(x a) b=x a$.

From (2), $x a^{\prime}=z$, whence by $6.37, x a=\dot{x}$. Hence $(x a) b=x$, whence by 6.26 ,

$$
x(a b)=x .
$$

From (3) and (5), by $6.27,6.26,6.25,6.32,6.31$,

$$
x=x x=[x(a b)]\left[x(a b)^{\prime}\right]=x\left[(a b)(a b)^{\prime}\right]=x z=z .
$$

6.40. $a b+a b^{\prime}=a$.

By $6.25,6.26,6.27,(a b) a=a(a b)=(a a) b=a b$, whence by 6.38 ,

$$
a b+a=a .
$$

From (1),

$$
a b^{\prime}+a=a .
$$

Hence $(a b+a)+\left(a b^{\prime}+a\right)=a+a$, whence by 6.25, 6.26, 6.27, $a+\left(a b+a b^{\prime}\right)=a$. But by $6.39, a\left(a b+a b^{\prime}\right)^{\prime}=z$, whence by $6.36, a+\left(a b+a b^{\prime}\right)=a b+a b^{\prime}$. Therefore $a b+a b^{\prime}=a$.

6.41. $\left(a^{\prime}+b^{\prime}\right)^{\prime}+\left(a^{\prime}+b\right)^{\prime}=a$. (From 6.40 , by 6.24 and 6.23 .)

The proof of the equivalence of the sixth set and the fourth set is thus complete; Theorems $6.1,6.2,6.11,6.12,6.13$, and 6.41 show that all the postulates of the fourth set are deducible from the sixth set. 
INDEPENDENCE PROOFS FOR THE SIXTH SET

We first give three examples for the independence of Postulate 6.8.

Example 6.8 (1). $K=$ three elements, 0, 1, 2; with $a+b$ and $a^{\prime}$ given by the table.

\begin{tabular}{|c|c|c|c|c|}
\hline \pm & 0 & 1 & 2 & \\
\hline 0 & 0 & 1 & 2 & 1 \\
\hline 1 & 1 & 1 & 1 & 0 \\
\hline 2 & 2 & 1 & 2 & 1 \\
\hline
\end{tabular}

This system $\left(K,+,{ }^{\prime}\right)$ has all the properties called for by Postulates 6.1-6.7, with the subclass $T$ consisting of the single element 1 . The system fails on 6.8 , since $2^{\prime}+0=1$ and $0^{\prime}+2=1$, but not $2=0$. It is interesting to note that $a^{\prime \prime \prime}+a$ in $T$ and $a^{\prime}+a^{\prime \prime}$ in $T$ both hold, but $a^{\prime \prime}=a$ is not true when $a=2$. We note also that $a+b=b+a$ and $(a+b)+c=a+(b+c)$ and $a+a=a$; further, if $a+b$ is in $T$ then $a$ is in $T$ or $b$ is in $T$.

Example 6.8 (2). $K=$ five elements. (This example was suggested to me, in another connection, by Dr. K. E. Rosinger.)

\begin{tabular}{c||ccccc||c}
+ & 0 & 1 & 2 & 3 & 4 \\
\hline 0 & $=0$ & 1 & 2 & 3 & 4 & \\
1 & 1 & 1 & 1 & 1 & 1 & \\
2 & 2 & 1 & 2 & 3 & 1 & \\
3 & 3 & 1 & 3 & 3 & 1 & 4 \\
4 & 4 & 1 & 1 & 1 & 4 & 4 \\
4
\end{tabular}

Postulates 6.1-6.7 hold, with the subclass $T$ consisting of the single element 1. Postulate 6.8 fails, since $2^{\prime}+3=1$ and $3^{\prime}+2=1$, but not $2=3$. Here $a+b=b+a$ and $(a+b)+c=a+(b+c)$ and $a+a=a$ are always true, but not $a^{\prime \prime}=a$. Further, $a+b$ can equal 1 when neither $a=1$ nor $b=1$.

Example 6.8 (3). $K=$ six elements. (This example was suggested to me by Mr. P. Henle.)

\begin{tabular}{c||cccccc||c}
+ & 0 & 1 & 2 & 3 & 4 & 5 \\
\hline 0 \\
1
\end{tabular}


Postulates 6.1-6.7 will be found to hold, with the subclass $T$ consisting of the single element 1 . Postulate 6.8 fails, since $2^{\prime}+5=1$ and $5^{\prime}+2=1$, but not $2=5$. We note that $a^{\prime \prime}=a$ holds. Also, $(a+b)^{\prime}+(b+a)=1$, but not $a+b=b+a$. Also, $(a+a)^{\prime}+a=1$ and $a^{\prime}+(a+a)=1$, but not $a+a=a$.

Obvious examples for Postulate 6.1 and 6.2 are the following, in which $x$ is any object not in the class $K$.

Example 6.1

\begin{tabular}{c||cccc||c}
+ & 0 & 1 & 2 & 3 \\
\hline \hline 0 & 0 & 1 & 2 & 3 & \\
1 & 1 & 1 & 1 & 1 & 1 \\
2 & 2 & 1 & 2 & $x$ & 0 \\
3 & 3 & 1 & $x$ & 3 & 3 \\
& & & \\
& 0
\end{tabular}

Example 6.2

\begin{tabular}{c||cccc||c}
+ & 0 & 1 & 2 & 3 \\
\hline \hline 0 \\
1
\end{tabular}

The remaining examples (for 6.3-6.7) I take from a recent paper by P. Henle (The independence of the postulates of logic, Bulletin of the American Mathematical Society, 1932).

Example 6.3

$$
\begin{array}{c||cc||c}
+ & 0 & 1 \\
\hline \hline 0 & 1 & 1 & \\
1 & 1 & 1 & 0
\end{array}
$$

Example 6.4

\begin{tabular}{c||ccc||c}
+ & 0 & 1 & 2 \\
\hline \hline 0 & 0 & 1 & 2 \\
1 & 1 & 1 & 1 & \\
2 & 2 & 1 & 1
\end{tabular} \mid $\begin{aligned} & 1 \\
& 0 \\
& 2\end{aligned}$

Example 6.5

\begin{tabular}{c||cccc||c}
+ & 0 & 1 & 2 & 3 & \multicolumn{1}{c}{} \\
\hline \hline 0 & $\overline{0}$ & 1 & 0 & 0 & 1 \\
1 & 1 & 1 & 1 & 1 & 0 \\
2 & 0 & 1 & 0 & 1 & 3 \\
3 & 0 & 1 & 1 & 0 & 2
\end{tabular}

Example 6.6

\begin{tabular}{c||cccc||c}
+ & 0 & 1 & 2 & 3 \\
\hline 0 & 0 & 1 & 2 & 3 & \multicolumn{1}{c}{} \\
1 & 1 & 1 & 1 & 1 & 1 \\
2 & 2 & 1 & 2 & 1 & 0 \\
3 & 0 & 1 & 1 & 3 & 2 \\
& &
\end{tabular}

Example 6.7

\begin{tabular}{c||cccccc||c}
+ & 0 & 1 & 2 & 3 & 4 & 5 \\
\hline 0 & 0 & 1 & 2 & 3 & 4 & 5 & \\
1 & 1 & 1 & 1 & 1 & 1 & 1 & \\
2 & 2 & 1 & 2 & 1 & 1 & 1 & 1 \\
3 & 3 & 1 & 1 & 3 & 4 & 5 & \\
4 & 4 & 1 & 1 & 4 & 4 & 1 & \\
5 & 5 & 1 & 1 & 5 & 1 & 5
\end{tabular}

The following unsolved problem may be noted. If 6.8 were replaced by the same postulate without the qualifying clause (call it $6.8 \mathrm{a}$ ), then the independence of 6.3 would become an open question (since the present Example 6.3 does not satisfy $6.8 \mathrm{a}$ ). 


\section{APPENDIX I}

The connection between Boolean algebra and the Principia

In order to establish the connection between Boolean algebra and the system set forth in Section A of the Principia, we first quote the following propositions verbatim from the second edition of the Principia.

$* 1.71$. If $p$ and $q$ are elementary propositions, $p \vee q$ is an elementary proposition.

$* 1.7$. If $p$ is an elementary proposition, $\sim p$ is an elementary proposition.

*4.31. $\vdash: p \vee q \cdot \equiv \cdot q \vee p$.

*4.33. $\vdash:(p \vee q) \vee r \cdot \equiv \cdot p \vee(q \vee r)$.

*4.25. $\vdash: p \cdot \equiv \cdot p \vee p$.

*4.5. $\vdash: p \cdot q \cdot \equiv \cdot \sim(\sim p \vee \sim q)$.

$* 4.42$. $\vdash: \cdot p \cdot \equiv: p \cdot q \cdot v \cdot p \cdot \sim q$.

If now we call the class of "elementary propositions" the class $K$, and write $p+q$ for $p \vee q$, and $p^{\prime}$ for $\sim p$, these propositions become the following:

$* 1.71$. If $p$ and $q$ are in the class $K$, then $p+q$ is in the class $K$.

*1.7. If $p$ is in the class $K$, then $p^{\prime}$ is in the class $K$.

*4.31. $p+q \equiv q+p$.

*4.33. $(p+q)+r \equiv p+(q+r)$.

*4.25. $p \equiv p+p$.

*4.5. $p q \equiv\left(p^{\prime}+q^{\prime}\right)^{\prime}$.

*4.42. $p \equiv p q+p q^{\prime}$.

But these propositions are precisely the same as the postulates of our fourth set for Boolean algebra, except that the sign $\equiv$ occurs in place of the sign $=$.

It remains, therefore, to examine the properties of the sign $\equiv$ as used in the Principia, in comparison with Postulates A, B, C, D governing the use of the sign $=$.

Here it is necessary to distinguish between the formal statements and the informal statements in the Principia. Among the formal statements we find

*4.2. . . $p \equiv p$, which is the same as Postulate A.

Another formal statement (in view of $* 4.01$ and $* 1.01$ ) is

*4.21. $\vdash:(p \equiv q)^{\prime} \cdot v \cdot(q \equiv p)$.

In accordance with the informal statement under $(6)$ in $* 1$, this formula means

$$
\text { " } p \equiv q \text { is false or } q \equiv p \text { is true," }
$$

and this in turn means

"If $p \equiv q$, then $q \equiv p, "$

which is the same as Postulate B. 
Among the informal statements we find under $* 4.22$ that " $\equiv$ " denotes a relation, namely the "relation of equivalence," and that "the relation of equivalence is reflexive $\left({ }^{*} 4.2\right)$, symmetrical $\left({ }^{*} 4.21\right)$ and transitive $\left({ }^{*} 4.22\right) . " \dagger$

We have already cited $* 4.2$ and $* 4.21$. In regard to $* 4.22$, the formal statement of this theorem (in view of *1.01) is

*4.22. $\vdash: \sim(p \equiv q \cdot q \equiv r) \cdot v \cdot(p \equiv r)$;

and in accordance with the informal statement under (6) in *1, this formula means " $p \equiv q \cdot q \equiv r$ is false or $p \equiv r$ is true." This in turn means

$$
\text { "If } p \equiv q \text { and } q \equiv r \text {, then } p \equiv r, "
$$

which is the same as Postulate $\mathrm{C}$.

Again, among the informal statements we find under $* 4.01$ the following:

"If $p \equiv q$, then $q$ may be substituted for $p$ without altering the truth-value of any function of $p$ which involves no primitive ideas except those enumerated in *1."

This comes to the same thing as our Postulate D.

Hence, if we accept the above mentioned informal statements as a valid part of the theory of the Principia, we have the following theorem:

TheOREM I. With respect to ( $K, \vee, \sim, \equiv)$, the informal system of the Principia is a Boolean algebra.

Here, from the abstract postulational point of view, $K$ is an undefined class; $\vee($ or + ) is an undefined binary operator; $\sim$ (or ') is an undefined unary operator; and $\equiv$ is an undefined relation. Concretely, $K$ may be interpreted as the class of "elementary functions"; $a \vee b$ as " $a$ or $b$ "; $\sim a$ as "not- $a$ "; and $a \equiv b$ as " $a$ equivalent to $b$. " But any other interpretation of the symbols $(K, \vee, \sim, \equiv)$ which satisfies the rules laid down would be a valid example of the system.

One further question arises. Since the number of elements in a Boolean algebra may be any power of 2 , it is interesting to inquire whether there is anything in the Principia which restricts the number of elements.

Among the formal statements we find

*5.15. $\vdash: p \equiv q \cdot \vee \cdot p \equiv \sim q$;

and according to the informal statement under (6) in *1, this formula means

"Either $p \equiv q$ is true or $p \equiv \sim q$ is true."

That is, if $q$ is any particular element of the class $K$, then every other element must be equivalent either to $q$ or to $q^{\prime}$; so that there are only two non-

$\dagger$ In the formal part of the Principia there is a different definition of $\equiv$, which does not concern us here. 
equivalent elements in the system. Hence, if we accept the informal as well as the formal statements, we have

Theorem II. With respect to $(K, \vee, \sim, \equiv)$, the informal system of the Principia is a Boolean algebra containing only two non-equivalent elements.

\section{APPENDIX II}

A set of independent postulates for Principia Mathematica

This appendix contains a revision of the primitive propositions of the Principia (Section A), without making use of the equality sign, or the Boolean notation " $=1$." (Compare Bernstein's papers of 1931 and 1932.)

The primitive ideas in this theory are four in number:

$K=$ an undefined class of elements, $a, b, c, \cdots$ ( $K$ being interpretable as the class of "propositions");

$T=$ an undefined subclass in $K$ ( $T$ being interpretable as the subclass of "true" propositions, indicated in the Principia by the assertion sign $\vdash$ );

$a+b=$ the result of an undefined binary operation $(a+b$ being interpretable as " $a$ or $b$," denoted in the Principia by $a \vee b)$;

$a^{\prime}=$ the result of an undefined unary operation ( $a^{\prime}$ being interpretable as "not- $a$," denoted in the Principia by $\sim a$ ).

The postulates here assumed are seven in number, the first five corresponding precisely to "formal," and the last two to "informal" statements in the Principia:

Postulate 1. If $a$ and $b$ are in $K$, then $a+b$ is in $\left.K .{ }^{*} 1.71\right]$

Postulate 2. If $a$ is in $K$, then $a^{\prime}$ is in $K$. [*1.7]

Postulate 3. If $a, b$, etc. are in $K$, then $b^{\prime}+(a+b)$ is in $T$. [*1.3]

Postulate 4. If $a, b$, etc. are in $K$, then $(a+b)^{\prime}+(b+a)$ is in $T$. [*1.4]

Postulate 5. If $a, b, c$, etc. are in $K$, then $\left(b^{\prime}+c\right)^{\prime}+\left[(a+b)^{\prime}+(a+c)\right]$ is in $\left.T .{ }^{*} 1.6\right]$

Postulate 6. If $a+b$ is in $T$, then at least one of the elements $a$ and $b$ is in $T$.

Postulate 7. If $a^{\prime}$ is in $T$, then $a$ is not in $T$.

From these postulates the following propositions are deducible as theorems. $\dagger$

† The proof of 8 follows at once from 6 .

The proof of 9 depends on the following lemmas:

(a) If $a+b$ is in $T$, then $b+a$ is in $T$. (By 4, 6, 7.)

(b) If $b$ is in $T$, then $a+b$ is in $T$. (By $3,6,7$.)

(c) If $a$ is in $T$, then $a+b$ is in T. (By (a) and (b).)

(d) If $a$ is not in $T$ and $b$ is not in $T$, then $a+b$ is not in $T$. (By 6.)

(e) If $a+b$ is not in T, then $a$ is not in $T$ and $b$ is not in $T$. (By (b) and (c).)

If 9 were false, we should have, by (e) and (d), $(a+a)^{\prime}+(a+a)$ not in $T$, contrary to 4 . The proof of 10 (due to Bernays in 1926) is given in 6.10 above. 
8. If $a$ is in $T$ and $a^{\prime}+b$ is in $T$, then $b$ is in $T$. [*1.1]

9. If $a, a+a$, etc. are in $K$, then $(a+a)^{\prime}+a$ is in T. [*1.2]

10. If $a, b, c$, etc. are in $K$, then $[a+(b+c)]^{\prime}+[b+(a+c)]$ is in $T .\left[{ }^{*} 1.5\right]$

Any system $\left(K, T,+,{ }^{\prime}\right)$ which satisfies Postulates 1-7 may be called an "informal Principia system," since all the propositions, both "formal" and "informal," in Section A of the Principia are deducible from these seven postulates.

In regard to the definitions of $a \supset b$ and $a \equiv b$, the "formal" and "informal" statements in the Principia are not in precise agreement. In the "formal" part (see $*^{*} .01$ and $* 4.01$ ), $a \supset b$ and $a \equiv b$ are defined as elements determined by $a$ and $b$ (analogous to $a+b$ ). But in the "informal" part (and in practically all common usage) the symbols $\supset$ and $\equiv$ are used to indicate relations between the two elements. These two usages may be reconciled by defining the symbols $a \supset b$ and $a \equiv b$ as elements, and the words " $a$ implies $b$ " and " $a$ is equivalent to $b$ " as statements about these elements.

11a. Definition. $a \supset b$ means $a^{\prime}+b$. [ $\left.{ }^{*} 1.01\right]$

11b. Definition. ( $a$ implies $b)$ means $\left(a^{\prime}+b\right.$ is in $\left.T\right)$.

That is, " $a$ implies $b$ " means that the element $a \supset b$ is in $T$.

12a. Definition. $a \equiv b$ means $\left[\left(a^{\prime}+b\right)^{\prime}+\left(b^{\prime}+a\right)^{\prime}\right]^{\prime}$. [ $\left.{ }^{*} 4.01\right]$ $T\}$.

12b. Definition. ( $a$ is equivalent to $b)$ means $\left\{\left[\left(a^{\prime}+b\right)^{\prime}+\left(b^{\prime}+a\right)^{\prime}\right]^{\prime}\right.$ is in

That is, " $a$ is equivalent to $b$ " means that the element $a \equiv b$ is in $T$.

From Postulates 1-7, with the aid of these definitions, the following muchdiscussed theorems are deducible:

13. If $a$ is in $K$, and $b$ is in $T$, then $a \supset b$ is in $T$. [*2.02]

That is, "a true proposition is implied by any proposition."

14. If $a$ is not in $T$ and $b$ is in $K$, then $a \supset b$ is in $T$. [*2.21]

That is, "a false proposition implies any proposition."

15. If $a$ is in $T$ and $b$ is in $T$, then $a \equiv b$ is in $T$. [*5.1]

That is, "two propositions are equivalent if they are both true."

16. If $a$ is not in $T$ and $b$ is not in $T$, then $a \equiv b$ is in $T$. [*5.21]

That is, "two propositions are equivalent if they are both false."

Hence an "informal Principia system," as above defined, contains only two "non-equivalent" elements.

17. If $a^{\prime}+(b+c)$ is in $T$, then $\left(a^{\prime}+b\right)+\left(a^{\prime}+c\right)$ is in $T$. [*4.78]

18. If $a^{\prime}+(b+c)$ is in $T$, then at least one of the elements $a^{\prime}+b$ and $a^{\prime}+c$ is in $T$.

That is, if $a$ implies $b+c$, then $a$ implies $b$ or $a$ implies $c$.

It can be shown, by definite examples of systems $\left(K, T,+,{ }^{\prime}\right)$, that Theorems 14, 16, and 18 cannot be deduced from the "formal" part of the 
Principia (1-5, 8-10), without the aid of the "informal" statement here listed as Postulate 6. (See Example 6, below.)

The consistency of Postulates 1-7 is shown by any one of the three following examples of systems $\left(K, T,+,{ }^{\prime}\right)$ in which all seven postulates are satisfied.

Example 0.1.

$K=\mathrm{a}$ class of four numbers, say $0,1,2,3$;

$T=$ the class containing the two numbers 1 and 3 ;

$a+b=$ the number given by the table;

$a^{\prime}=$ the number given by the table.

\begin{tabular}{|c|c|c|c|c|c|}
\hline+ & 0 & 1 & 2 & 3 & \\
\hline 0 & 0 & 1 & 2 & 3 & 1 \\
\hline 1 & 1 & 1 & 1 & 1 & 0 \\
\hline 2 & 2 & 1 & 2 & 1 & 3 \\
\hline 3 & 3 & 1 & 1 & 3 & 2 \\
\hline
\end{tabular}

Example 0.2.

$K=\mathrm{a}$ class of five numbers, say $2,3,4,8,9$;

$T=$ the class containing the three numbers $2,3,4$;

$a+b$ and $a^{\prime}=$ the numbers given by the table.

\begin{tabular}{|c|c|c|c|c|c|c|}
\hline+ & 2 & 3 & 4 & 8 & 9 & \\
\hline 2 & & 2 & 3 & & 2 & 8 \\
\hline 3 & 2 & 2 & 4 & 4 & 2 & 8 \\
\hline 4 & 3 & 4 & 3 & 4 & 2 & 9 \\
\hline 8 & 4 & 4 & 4 & 8 & 9 & 2 \\
\hline 9 & 3 & 3 & 3 & 9 & 9 & 3 \\
\hline
\end{tabular}

(Here the selection and arrangement of elements in each of the four compartments of the table is arbitrary, provided the two groups $2,3,4$ and 8, 9, are kept separate.)

Example 0.3.

$K=\mathbf{a}$ class of two numbers, 0,1 ;

$T=$ the class containing the single element 1 ;

$a+b$ and $a^{\prime}=$ the numbers given by the table.

\begin{tabular}{c||cc||c}
+ & 0 & 1 \\
\hline \hline 0 & 0 & 1 & \multicolumn{1}{c}{} \\
1 & 1 & 1 & 0
\end{tabular}


The independence of Postulates 1-7 is shown by the following examples of systems $\left(K, T,+,{ }^{\prime}\right)$, each of which violates the like-numbered postulate and satisfies all the other six.

Example 1. Same as Example 6.1, with $T=1$.

Example 2. Same as Example 6.2, with $T=1,3$.

Example 3. Same as Example 6.5, with $T=1,3$.

Example 4. Same as Example 6.6, with $T=1,3$.

Example 5. Same as Example 4.6, with $T=1,2,3,4$.

Example 6. Same as Example 0.1, above, with $T=1$ instead of $T=1,3$.

Example 7. Same as Example 0.1, above, with $T=0,1,2,3$ instead of $T \doteq 1,3$.

The last two examples satisfy not only Postulates 1-5, but also Theorems 8, 9, and 10; so that Postulates 6 and 7 are not deducible from the "formal" part of the Principia. This fact is of fundamental importance in any discussion of the adequacy of the "theory of deduction" as set forth in the formal part of the Principia.

HaRVARD UNIVERSITY,

Cambridge, Mass. 